\title{
TRPM channels mediate learned pathogen avoidance following intestinal distention
}

\author{
Adam Filipowicz and Alejandro Aballay* \\ Department of Molecular Microbiology \& Immunology, Oregon Health \& Science University, \\ Portland, OR 97239, USA \\ *Corresponding author. Email: aballay@ohsu.edu
}

\begin{abstract}
Upon exposure to harmful microorganisms, hosts engage in protective molecular and behavioral immune responses, both of which are ultimately regulated by the nervous system. Using the nematode Caenorhabditis elegans, we show that ingestion of $E$. faecalis leads to a fast pathogen avoidance behavior that results in aversive learning. We have identified multiple sensory mechanisms involved in the regulation of avoidance of $E$. faecalis, including the GPCR NPR-1-dependent oxygen-sensing pathway, an ASE neuron-dependent pathway, and an AWB and AWC neuron-dependent pathway. Colonization of the anterior part of the intestine by $E$. faecalis leads to AWB and AWC mediated olfactory aversive learning. Finally, two transient receptor potential melastatin (TRPM) channels, GON-2 and GTL-2, mediate this newly described rapid pathogen avoidance. These results suggest a mechanism by which TRPM channels may sense the intestinal distension caused by bacterial colonization to elicit pathogen avoidance and aversive learning by detecting changes in host physiology.
\end{abstract}




\section{INTRODUCTION}

In order to survive, animals have evolved mechanisms to detect and avoid harmful organisms, including pathogenic bacteria (Kavaliers et al., 2019; Medzhitov et al., 2012). Understanding this complex survival mechanism will ultimately take an interdisciplinary approach. Genetically tractable organisms such as Caenorhabditis elegans and Drosophila melanogaster have proven to be quite useful in uncovering the molecular and cellular components of pathogen avoidance. In response to pathogen exposure, these relatively simple animals are capable of eliciting avoidance behaviors (Anderson and McMullan, 2018; Cao et al., 2017; Meisel and Kim, 2014; Meisel et al., 2014; Pradel et al., 2007; Reddy et al., 2009; Singh and Aballay, 2019a, 2019b; Soldano et al., 2016; Stensmyr et al., 2012; Styer et al., 2008; Tran et al., 2017; Zhang et al., 2005) along with the activation of well-described microbicidal innate immune pathways (Engelmann et al., 2011; Irazoqui et al., 2010; Singh and Aballay, 2019b; Styer et al., 2008), both of which are regulated by the nervous system (Singh and Aballay, 2020; Wani et al., 2020).

It was recently proposed that bacterial colonization causes a distension of the $C$. elegans intestine that may be used as a general mechanism to activate immune pathways and pathogen avoidance (Kumar et al., 2019; Singh and Aballay, 2019a, 2019b). However, the mechanism by which the nematode senses intestinal distension remains unclear. One possibility is that mechanoreceptors on intestinal cells sense the pressure caused by distention. While no mechanoreceptors have been found to be functional in the $C$. elegans intestine, it does express transient receptor (TRP) channels which, in addition to other roles, could act as mechanoreceptors (Xiao and Xu, 2011). While the TRP vanilloid (TRPV) channel genes ocr-2 and osm-9 are known to inhibit avoidance of the pathogen Pseudomonas aeruginosa (Singh and Aballay, 2019a), this inhibition is through their role on hyperoxia avoidance rather than direct sensing of bacteria. Moreover, they are not expressed in the $C$. elegans intestine. One TRP channel that is expressed in the $C$. elegans intestine is the thermosensitive channel TRPA-1 (Xiao et al., 2013), TRPA-1 also plays a role in sensory neuron-mediated nose-touch responses (Kindt et al., 2007). Interestingly, the Drosophila homolog of this channel was shown to be involved in avoidance of bacterial lipopolysaccharide (LPS), though activity of this channel was localized to gustatory neurons (Soldano et al., 2016). The mammalian digestive system expresses many TRP channels, including members of the TRPA, TRP melastatin (TRPM) and TRPV families, that play a role in processes raging from pain to ion homeostasis (Holzer, 2011). Because nearly all of these channels are conserved across species, identification of their role in pathogen avoidance in $C$. elegans is likely to improve our understanding of pathogen avoidance in a range of organisms.

Here, we present evidence that $C$. elegans avoids Enterococcus faecalis faster than $P$. aeruginosa. In contrast to the $P$. aeruginosa-mediated avoidance that requires small RNAs (sRNAs) (Kaletsky et al., 2020), E. faecalis does not depend on sRNAs, nor does it require ASI 
neurons, full bacterial virulence, or innate immune activation. Instead, avoidance correlates with a rapid expansion of the anterior part of the intestine. This avoidance behavior requires an NPR-1-mediated hyperoxia avoidance process as well as the ASE, AWB, and AWC chemosensory neurons. These latter two neuron pairs regulate an olfactory aversive learning process that underpins avoidance of $E$. faecalis. Finally, we identified two novel regulators of intestinal distention-induced pathogen avoidance: the TRP melastatin (TRPM) channels GON-2 and GTL-2.

\section{RESULTS}

\section{E. faecalis elicits fast avoidance in C. elegans}

C. elegans is a free-living nematode that feeds on organic material rich in microorganisms, and therefore frequently encounters pathogenic bacteria. It has developed behavioral strategies to minimize exposure to these threats. For example, it avoids the pathogenic $P$. aeruginosa bacteria slowly, taking between 12 to 24 hours to execute aerotactic and olfactory aversive learning processes depending on the bacterial growth conditions used (Singh and Aballay, 2019a; Zhang et al., 2005). In contrast, we previously reported that animals quickly avoid the lawns of the Gram-positive pathogens E. faecalis, E. faecium, and S. aureus (Singh and Aballay, 2019b). Examining this in more detail, we found that animals displayed strong, fast avoidance of monoaxenic lawns of E. faecalis, E. faecium, or S. aureus grown on brain heart infusion (BHI) media (Figure 1A, 1B, and Figure 1 - figure supplement 1). While population avoidance levels peaked at 4 hours for $E$. faecalis, wild-type animals first leave E. faecalis lawns at around 19 minutes on average (Figure $1 \mathrm{C}$ ). Interestingly, we observed animals frequently exiting and entering the bacterial lawns over time. Quantification of these events revealed a slight imbalance favoring exiting events as early as 1 hour, with this difference growing larger in the 3-4 hour window due to a decrease in the entry events (Figure 1D).

The fast exiting events induced by $E$. faecalis are reminiscent of a $C$. elegans rapid aversive behavior elicited by exposure to a dry-drop of dodecanoic acid, a toxin secreted by Streptomyces (Tran et al., 2017). However, implementing this dry-drop assay with $E$. faecalis or E. faecium did not induce an aversive response (Figure 1 - figure supplement 1 ), indicating that direct contact with live replicating bacteria is required for the rapid avoidance of $E$. faecalis or E. faecium. Rapid aversion alone also cannot explain the re-entry events. Instead, it is likely that there is a balance of attraction and aversion to $E$. faecalis that changes over the course of exposure. Thus, the animals learn to avoid E. faecalis quickly after exposure. This process likely involves sensory pathways, as animals with loss-of-function mutations in tax-2, a subunit of a cGMP-gated-ion-channel important for sensory neuron function (Coburn and Bargmann, 1996), show slowed avoidance (Figure $1 \mathrm{C}$ ). Because it has recently been suggested that the sensation of a bacterial sRNAs is at least partly responsible for learned $P$. aeruginosa avoidance (Kaletsky et al., 2020), we examined whether RNA from E. faecalis may also elicit avoidance. Unlike RNA 
from $P$. aeruginosa, RNA from $E$. faecalis fails to induce avoidance (Figure $1 \mathrm{E}$ ). The fact that exposure to bacterial sRNAs only accounts for $\sim 25 \%$ of the avoidance of $P$. aeruginosa, and that they have no role in avoidance of $E$. faecalis, suggests that another pathway is required to induce pathogen avoidance.

\section{Anterior intestinal distention elicits avoidance of $E$. faecalis independently of virulence}

Two other potential pathways for induction of avoidance are sensation of intestinal distention or bacterial metabolites. The bacterial metabolite serrawettin W2 is responsible for the avoidance of S. marcescens (Pradel et al., 2007). Even though secondary metabolites phenazine-1-carboxamide and pyochelin produced by $P$. aeruginosa are insufficient for the elicitation of pathogen avoidance (Singh and Aballay, 2019a), they are sensed by $C$. elegans as they elicit induction of DAF-7/TGF- $\beta$ in ASJ neurons (Meisel et al., 2014). However, E. faecalis does not increase expression of DAF-7 in the ASJ neurons (Meisel et al., 2014), further strengthening the idea that direct contact with this pathogen rather than metabolite sensing induces avoidance.

To determine whether intestinal distention plays a role in avoidance of $E$. faecalis, we measured the diameter of the intestinal lumen of animals fed either $E$. coli, P. aeruginosa, or $E$. faecalis grown on BHI. There was significant expansion of the anterior part of the intestine of animals feeding on $E$. faecalis compared to $E$. coli even at 1 hour, with distention persisting at 4 hours (Figure 2A and B). The medial part of the intestine was not significantly bloated at 1 hour compared to animals fed $E$. coli, but was bloated at 2 and 4 hours. The anterior part of the intestine was slightly bloated in animals feeding on $P$. aeruginosa compared to $E$. coli at 4 hours, but was otherwise not bloated. Both parts of the intestine, but especially the anterior portion, were filled with live $E$. faecalis, as determined through the use of an $E$. faecalis strain expressing GFP, while the intestine of animals fed $E$. coli or $P$. aeruginosa expressing GFP were relatively empty and not distended (Figure $2 \mathrm{C}$ ). It is therefore likely that fast accumulation of $E$. faecalis in the anterior intestine induces an aversive response to the pathogen, leading to avoidance. RNA interference (RNAi)-mediated knockdown of nol-6, a nucleolar RNA-associated protein, reduces colonization and distention of the intestine by microbial pathogens (Fuhrman et al., 2009). Furthermore, knockdown of nol-6 delays avoidance of $P$. aeruginosa by delaying intestinal distention (Singh and Aballay, 2019a; Figure 2 - figure supplement 1). Therefore, we tested both nol-6 RNAi and a nol-6 loss-of-function (If) mutant (nol-6(ac1)) for avoidance of $E$. faecalis. Avoidance for both the mutant and nol-6 RNAi remained at wild-type levels (Figure 2D). Interestingly, while anterior intestinal distension was observed in both nol-6(ac1) and nol-6 RNAi animals fed $E$. faecalis at 4 hours, the middle part of the intestine did not display significant distention (Figure 2E, F, and Figure 2 - figure supplement 1 ). This suggests that the anterior, but not medial, intestinal distention triggers avoidance of $E$. faecalis. 
To determine whether bacterial virulence is required for anterior intestine distention induced avoidance of $E$. faecalis, we used a strain of $E$. faecalis lacking the $f s r B$ gene (E. faecalis $\Delta f s r B)$, a gene important for quorum sensing, which displays significantly reduced virulence across multiple animal models (Garsin et al., 2001; Mylonakis et al., 2002; Sifri et al., 2002). Surprisingly, this avirulent strain also elicits avoidance (Figure 3A). Lending further evidence to the notion that virulence plays no role in avoidance, $E$. faecium, a related enterococcal species that is non-pathogenic to C. elegans (Garsin et al., 2001; Yuen and Ausubel, 2018), also elicits avoidance (Figure 1B). While E. faecium is non-pathogenic to $C$. elegans it does cause an innate immune response (Yuen and Ausubel, 2018), raising the possibility that an early immune response may be responsible for alerting the animal to avoid E. faecalis and related species upon ingestion.

Therefore, we examined the in vivo expression of two immune pathway markers, clec60 (Irazoqui et al., 2010; Yuen and Ausubel, 2018) and ilys-3 (Gravato-Nobre et al., 2016; Irazoqui et al., 2010; Yuen and Ausubel, 2018), to determine whether an immune response was mounted at these early timepoints. While a later immune response could be observed, there was no evidence of an immune response at timepoints where early avoidance of $E$. faecalis is observed (Figure 3B-D). As shown in Figure 3E, E. faecalis $\Delta f s r B$ induces anterior intestinal distension. Altogether, these results suggest that virulence and immune pathway activation is not necessary for anterior distension and avoidance of $E$. faecalis, differentiating this avoidance mechanism further from that induced by $P$. aeruginosa, which requires virulence and intestinewide distension (Kumar et al., 2019; Singh and Aballay, 2019a, 2019b).

\section{NPR-1 and TAX-2/4 pathways regulate avoidance of $E$. faecalis and $P$. aeruginosa}

NPR-1, known to play a role in C. elegans survival and behavior on pathogens (Meisel and Kim, 2014; Reddy et al., 2009; Styer et al., 2008), is responsible for the intestinal distention induced avoidance behavior on $P$. aeruginosa. We hypothesized that NPR-1 would also play a role in avoidance of $E$. faecalis. As a first step to test this, we measured avoidance of $E$. faecalis in two $n p r-1$ (If) mutants and found that both displayed decreased avoidance compared to wild-type animals (Figure 4A).

Inhibition of NPR-1 elicits avoidance of high oxygen (de Bono and Bargmann, 1998; Chang et al., 2006; Rogers et al., 2003), resulting in suppression of $P$. aeruginosa avoidance (Reddy et al., 2009; Styer et al., 2008), as the bacterial lawns have low oxygen due to microbial metabolism (Reddy et al., 2011). To test whether that is the case with avoidance of $E$. faecalis, wild-type and npr-1 (If) animals were placed into hypoxia (8\% oxygen) chambers and scored for avoidance. In this low oxygen environment, both npr-1(ad609) and npr-1(ok1447) displayed wild-type levels of avoidance (Figure 4B). Furthermore, when animals were taken out of the low oxygen environment and allowed to roam at atmospheric oxygen levels for one-hour, wild-type 
animals remained off the $E$. faecalis lawns while both $n p r-1(I f)$ strains migrated back onto the lawns (Figure 4C), indicating that hyperoxia avoidance overrides pathogen avoidance in $n p r-1(I f)$ animals.

Hyperoxia avoidance in $n p r-1(I f)$ animals requires the transient receptor potential channel vanilloid (TRPV) genes ocr-2 and osm-9 (de Bono et al., 2002; Chang et al., 2006; Singh and Aballay, 2019a). Loss-of-function mutations in these genes results in decreased hyperoxia avoidance, and should thus lead to increased pathogen avoidance. Indeed, while loss-offunction mutants in both genes displayed avoidance levels similar to wild-type animals at 4 hours (Figure 4 -supplement 1 ), their rate of avoidance was faster than that of wild-type animals (Figure 4 - supplement 1 ). Hyperoxia avoidance of $n p r-1($ If) nematodes also depends on functional gcy-35, tax-2, and tax-4 genes (Chang et al., 2006; Gray et al., 2004). GCY-35 is a soluble guanylyl cyclase (sGC) that binds directly to molecular oxygen (Cheung et al., 2004) while TAX-2 and TAX-4 are two subunits of a cGMP-gated-ion-channel (Coburn and Bargmann, 1996) thought to act downstream of GCY-35 (Coates and de Bono, 2002; Gray et al., 2004). Through the activity of GCY-35 and TAX-2/TAX-4, the sensory neurons AQR, PQR, and URX drive avoidance of high oxygen. We tested double loss-of-function mutants of gcy-35, tax-2, or tax-4 and $n p r-1$, hypothesizing that $g c y-35, \operatorname{tax}-2$, and tax-4 mutations would suppress the lack of pathogen avoidance of $n p r-1$ (ad609) animals. While this was indeed the case for gcy-35 mutation, tax-2 and tax-4 mutations failed to suppress the lack of avoidance of $E$. faecalis exhibited by $n p r-1$ (ad609) animals (Figure 4D). These results suggest that TAX-2 and TAX-4 function in neurons other than NPR-1-expresing cells to mediate pathogen avoidance.

We next tested the role of TAX-2 and TAX-4 in the NPR-1-mediated avoidance of $P$. aeruginosa using double mutants for tax-2 or tax-4 and npr-1. Surprisingly, and in contrast to avoidance of $E$. faecalis, tax-2 but not tax-4 mutation suppressed the avoidance of $P$. aeruginosa of $n p r-1$ (ad609) animals (Figure 4D). The discrepancy between the double mutants is that the tax-2(p694) allele used only affects a subset of the tax-2 expressing neurons (Harris et al., 2014). The tax-4(p678) allele, in contrast, affects all tax-4-expressing neurons (Figure 4E). Thus, the neurons affected by the tax-4(p678) allele, but spared by the tax-2(p694) allele, most likely play a role in avoidance of $P$. aeruginosa independent from the NPR-1 pathway. The results from the $E$. faecalis experiments, on the other hand, suggest that the tax-2(p694) allele covers additional neurons that regulate avoidance of that bacteria. To confirm this, we used single loss-of-function mutants for $\operatorname{tax}-2$ and tax-4, including a strain carrying an allele that affects expression in all tax-2 expressing neurons (tax-2(p671)). We indeed found that all strains tested displayed decreased avoidance of $E$. faecalis compared to wild-type animals, while only tax-2(p671) and tax-4(p678) mutants displayed decreased avoidance of $P$. aeruginosa (Figure $4 \mathrm{~F})$.

\section{ASE, AWB, and AWC neurons mediate avoidance of $E$. faecalis}


We turned our attention to uncovering the specific neurons in the TAX-2/TAX-4-dependent pathway that regulate avoidance of both $E$. faecalis and $P$. aeruginosa. To identify the neuron(s) responsible for $E$. faecalis avoidance, we employed two strategies. First, we asked whether tax2 expression in individual neurons would rescue the lack of pathogen avoidance of tax-2(p694) animals. This revealed that tax-2 expression in ASE neurons was sufficient for pathogen avoidance, while tax-2 expression in the AQR, PQR, and URX neurons, had no effect (Figure 5A). Second, we used animals lacking ASE neurons and the individual AWB, AWC, and ASI neurons, which are spared by the tax-2(p694) allele. The results confirmed the involvement of ASE neurons in E. faecalis avoidance and showed that AWB and AWC neurons are also necessary (Figure 5B).

For avoidance of $P$. aeruginosa, we also employed the genetic ablation strategy, which revealed the requirement of $A W B$ and $A W C$ neurons (Figure $5 B$ ). Confirming the results of the tax-2 experiments, ASE neurons were not required for avoidance of $P$. aeruginosa. We also confirmed a previous report that ASI neurons, but not ASH neurons, are required for avoidance of $P$. aeruginosa (Cao et al., 2017), while showing that neither is required for avoidance of $E$. faecalis (Figure 5B). Using a two-choice assay where, unlike the pathogen avoidance assay that only uses $P$. aeruginosa, animals are given a choice between $E$. coli and $P$. aeruginosa, it was shown that ASI neurons respond to $P$. aeruginosa RNAs by eliciting avoidance (Kaletsky et al., 2020). Using both avoidance and choice assays, we found that animals lacking ASI neurons fail to avoid $P$. aeruginosa after total RNA exposure (Figure 5C, D). These results are consistent with the idea that ASI neurons are capable of sensing $P$. aeruginosa RNAs and that $E$. faecalis RNAs do not induce avoidance (Figure $1 \mathrm{E}$ ). Altogether, these results suggest that regulation of behavioral immunity is pathogen-specific (Figure 5E).

\section{AWB and AWC neurons are necessary for aversive olfactory learning following ingestion of $E$. faecalis}

Our data suggested that avoidance of $E$. faecalis represented an aversive learning process (Figure 1D), but we tested this directly. AWB and AWC neurons are known to play a role in odor preference and olfactory aversive learning in $C$. elegans in the context of $P$. aeruginosa (Ha et al., 2010; Harris et al., 2014). Because AWB and AWC neurons are also involved in avoidance of E. faecalis, we hypothesized that odor preference and olfactory aversive learning may also play a role in avoidance of $E$. faecalis. We first set out to establish the naïve preference of $C$. elegans when given the choice between E. coli and E. faecalis. We used a two-choice assay in which animals were able to roam for one hour on test plates containing $E$. coli and $E$. faecalis lawns on opposite sides of the plates before scoring animals based on lawn occupancy. Under this condition, animals showed a preference for $E$. coli over $E$. faecalis; however, if animals were instead paralyzed upon approaching the lawns, they displayed a preference for $E$. faecalis (Figure 6A). We hypothesized that there is an initial attraction to E. faecalis that is quickly 
overcome after feeding on it, such that animals go towards the familiar E. coli lawn instead. Paralyzing animals on lawn arrival captures the initial choice, while allowing them to freely roam captures the aversive learning behavior. We further hypothesized that this attractionaversion dynamic was at least partly olfactory, based on neuron ablation results (Figure 5), and evidence that tax-2(If) leads to significantly delayed lawn exiting (Figure $1 \mathrm{C}$ ).

To directly test the idea of an initial odor attraction, we modified the choice assay by attaching plugs of agar with bacterial lawns on opposite sides of the lids of empty test plates (Figure 6B, schematic), preventing the animals to get in direct contact with the bacteria. We then placed the anesthetic sodium azide on the test plate surface underneath the bacteria plugs and placed animals on the center of the plate, allowing them to migrate towards one side or the other. This revealed a naïve preference for the odor of $E$. faecalis over E. coli (Figure 6B, left). Both tax-2(p671) and tax-4(p678) mutants displayed decreased naïve odor preferences but $\operatorname{tax}-2(p 694)$ did not. This result is consistent with the idea that the $\operatorname{tax}-2$ and $\operatorname{tax}-4$ expressing neurons AWB and AWC are responsible for establishing the naïve odor preference. Indeed, ablating them also resulted in a decreased preference (Figure 6B, left). We next asked whether trained animals that were allowed to ingest $E$. faecalis would switch their odor preference. The animals were trained by exposing them to $E$. faecalis for four hours using the same plate setup that had been used for the avoidance assays. Then, the trained animals were transferred to the lid-choice testing plates. A marked shift in preference was observed for trained wild-type animals that was not seen in tax-2(p671), tax-4(p678), or AWB and AWC ablated strains (Figure 6B, left). The tax-2(p694) mutants, on the other hand, behaved like wildtype animals. The learning index for each strain also highlights that aversive olfactory learning takes place in wild-type and tax-2(p694) animals but not in tax-2(p671) or tax-4(p678) mutants or AWB and AWC ablated strains (Figure 6B, right). Thus, the tax-2 and tax-4 expressing AWB and $A W C$ neurons are necessary both for establishing a naïve preference and for aversive olfactory learning following ingestion of $E$. faecalis over a period of four hours.

\section{The TRPM channels gon-2 and $g$ t/-2 are required for distention-induced pathogen avoidance}

It remains unclear how $C$. elegans senses intestinal bloating in order to execute pathogen avoidance, either for $P$. aeruginosa or E. faecalis. Because TRP channels could be mechanoreceptors (Xiao and $X u, 2011$ ) capable of sensing the intestinal distension caused by microbial colonization, we screened animals with loss-of-function mutations in 5 TRP-encoding genes for avoidance of $E$. faecalis and found that two members of the TRPM subfamily, gon-2 and $g t /-2$, negatively affected avoidance of $E$. faecalis (Figure 7 - supplement 1 ). GON-2 is expressed mainly in the gonad and intestine while GTL-2 is expressed primarily in the excretory system, hypodermis, and pharynx (Teramoto et al., 2005). Because gon-2 mutants are known to have abnormal gonad development, and the germline has previously been shown to be involved in pathogen avoidance and immunity (Kaletsky et al., 2020; Moore et al., 2019; 
TeKippe and Aballay, 2010), we tested whether the effect of gon-2(If) mutations on pathogen avoidance was due to perturbed gonad development. We took advantage of the fact that the Gon phenotype is temperature sensitive (high penetrance at $25^{\circ} \mathrm{C}$ and low penetrance at $15^{\circ} \mathrm{C}$ ) and compared avoidance results for both $E$. faecalis and $P$. aeruginosa at both temperatures. For $P$. aeruginosa, gon-2(If) animals raised at $25^{\circ} \mathrm{C}$ failed to avoid, while animals raised at $15^{\circ} \mathrm{C}$ avoided like wild-type animals (Figure 7A and B). For E. faecalis, gon-2(If) animals failed to avoid at both temperatures (Figure 7A and B), suggesting that the germline does not play a role in the control of avoidance of this pathogen. Furthermore, glp-1(e2141) mutants, which lack most germline cells due to defects in mitotic and meiotic division (Austin and Kimble, 1987; Crittenden et al., 1994), behave similarly to gon-2(lf) mutant animals on both pathogens (Figure $7 \mathrm{~A}$ and B). This suggests that gon-2(If) animals fail to avoid $P$. aeruginosa specifically because of a lack of gonad development, while for $E$. faecalis the effect on avoidance is independent of the germline. Unlike gon-2(If), gt/-2(n2618) animals failed to avoid E. faecalis but not $P$. aeruginosa (Figure 7A and B).

We hypothesized that the effect observed for gon-2(If) and $g t /-2(n 2618)$ animals was due to an inability to sense intestinal distention either directly or indirectly. It was previously shown that knockdown of aex-5 leads to defects in the defecation motor program and subsequent intestinal distention by the accumulation of bacteria, resulting in increased avoidance of even non-pathogenic $E$. coli (Singh and Aballay, 2019a). Interestingly, gon-2(If) mutants failed to exhibit such avoidance on E. coli HT115 after knockdown of aex-5, while gt/2(n2618) mutants behaved like wild-type animals (Figure 7C). These results are consistent with the different types of distention caused by $E$. faecalis and $P$. aeruginosa, with the former causing a more severe but localized distention of the anterior intestine and the latter causing a distention along the entire length of the intestine, which seems to require the germline to elicit avoidance. As shown in Figure 7D, both gon-2 and gt/-2 mutants suppressed the aex-5 knockdown avoidance phenotype on E. faecalis, suggesting that both TRPM channels are necessary for sensing the anterior intestinal distention caused by E. faecalis.

\section{DISCUSSION}

This study establishes that E. faecalis infection in C. elegans leads to anterior intestinal distention which results in a rapid pathogen avoidance behavior, regulated by NPR-1-mediated hyperoxia avoidance, TAX-2/4 expressing AWB, AWC, and ASE sensory neurons, and the TRPM channels GON-2 and GTL-2. In contrast, avoidance of $P$. aeruginosa uses different mechanisms that involve intestine-wide distention, germline mediated-signaling, and sensing of bacterial sRNAs via an ASI neuronal pathway (Figure 7E). Thus, bacterial context is important for the elicitation of pathogen avoidance behaviors. This context-dependence makes it likely that using C. elegans may lead to the discovery of new components of sensory pathways involved in pathogen avoidance and help to elucidate how physiological information from the site of 
infection is relayed to the nervous system. This was indeed highlighted by the identification of ASE neurons and GTL-2 as being necessary for avoidance of $E$. faecalis but not $P$. aeruginosa, and the requirement of the germline in avoidance of $P$. aeruginosa but not $E$. faecalis.

The decision to leave a bacterial lawn represents a dynamic balance between risk and reward for $C$. elegans, with feeding and food choice being critical for survival and propagation (Kiyama et al., 2012; Milward et al., 2011; Shtonda and Avery, 2006). Initially, the balance may be towards staying on the lawn, as it could be an area of high reward in the form of food. However, depending on the quality of the food, its availability, and its potential noxious qualities, the balance may swing towards bacterial avoidance, as the lawn becomes either an area of low reward (depleted or low-quality) or an area of high risk (pathogenic). The frequent entering and exiting events of animals on lawns of E. faecalis (Figure 1C and 1D) may be indicative of this dynamic balance. Multiple sensory inputs or physiological states such as oxygen level, odors, tastes, mechanosensation, pain, and hunger must be integrated in order to evaluate the environment and come to a decision. These cues are most likely integrated in the interneurons of $C$. elegans, which are downstream of the sensory neurons (Metaxakis et al., 2018; Summers et al., 2015; Wilson et al., 2017).

The involvement of ASE neurons, largely described as gustatory neurons, in the avoidance of $E$. faecalis (Figures $3 A$ and $B$ ) suggests that taste may play a role in pathogen avoidance in $C$. elegans in certain contexts. ASE neurons have also been shown to be $\mathrm{CO} 2$ sensors (Bretscher et al., 2011), and polymodal sensory neurons are common in C. elegans (Kaplan and Horvitz, 1993; Metaxakis et al., 2018), raising the possibility that ASE sensation of carbon dioxide, and not gustation, may elicit avoidance of $E$. faecalis. Additionally, a recent study implicated ASEL as a secondary sensory neuron in the detection of the food odor benzaldehyde (Leinwand et al., 2015). Interestingly, AWC neurons are also recruited into a saltsensing circuit via neuropeptide signaling from ASE neurons depending on salt concentration changes (Leinwand and Chalasani, 2013), leaving open the possibility that AWC neurons also play a role in avoidance of $E$. faecalis (Figures $3 A$ and $B$ ) not only through olfactory aversive learning (Figure $6 \mathrm{C}$ ) but through taste aversive learning. Further studies will need to be conducted to determine which possible sensory modality is important for ASE-dependent and AWC-dependent avoidance of $E$. faecalis. ASE neurons were also identified as part of the complex circuitry underlying the decision to leave a resource-depleted food patch, possibly via carbon dioxide sensing (Milward et al., 2011), suggesting an interesting link between pathogen avoidance and adaptive food leaving.

AWB and AWC involvement in odor preference and olfactory aversive learning is wellestablished in various contexts (Ha et al., 2010; Harris et al., 2014; Yoshida et al., 2012), and the present study extends this to $C$. elegans interactions with E. faecalis (Figure 6C) while providing direct evidence of their involvement in avoidance for both $E$. faecalis and $P$. aeruginosa (Figures 
$3 A$ and B). The olfactory circuitry in C. elegans has been described in detail (Chalasani et al., 2007; Leinwand et al., 2015). AWC neurons also communicate and receive feedback from other interneurons through the use of neuropeptides, such as the NLP-1-NPR-11-INS-1 feedback loop between AWC and AIA interneurons (Chalasani et al., 2010). The circuitry for olfactory aversive learning has also received extensive study in the context of $P$. aeruginosa with serotonin and various neuropeptides playing crucial roles in learning and foraging states (Chen et al., 2013; Fadda et al., 2020; Flavell et al., 2013; Ha et al., 2010; Harris et al., 2014; Zhang et al., 2005). Interestingly, both AWB and AWC neurons were previously shown to play a role in avoidance of normally attractive food following undernourishment (Olofsson, 2014), providing another link between pathogen avoidance behavior and foraging. Furthermore, in a recent study, the transcription factor Nrf2/skn-1 was shown to be required in the AIY interneuron for the integration of information from ASE and AWC neurons during foraging (Wilson et al., 2017). Whether this same AIY neuronal integration is required for pathogen avoidance remains to be seen. Previous work in Drosophila has also revealed olfactory-mediated avoidance of harmful microbes (Stensmyr et al., 2012), illustrating that similar mechanisms are at play across different species.

The discovery of gon- 2 and $g t /-2$ playing a role in pathogen avoidance is novel. The most well-known impact of loss of gon-2 function is severe impairment of gonadogenesis due to disruption of gonadal cell divisions (Sun and Lambie, 1997). This impairment is most likely responsible for the results observed for avoidance of $P$. aeruginosa (Figure 7A and $\mathrm{B}$ ), as the germline and associated tissues are necessary for this avoidance behavior (Kaletsky et al., 2020; Moore et al., 2019; TeKippe and Aballay, 2010). The results of the glp-1 experiments (Figure 7A and $B$ ) further support this idea. However, the germline seems to play no role in avoidance of $E$. faecalis, and therefore other functions of gon-2 and gt/-2 may be responsible. The germline plays a role in a transgenerational learned avoidance of $P$. aeruginosa (Moore et al., 2019), but the lack of involvement of the germline (Figure 7A and B), bacterial sRNAs (Figure 3C), and ASI neurons (Figure $3 B$ ) in avoidance of $E$. faecalis suggest that such a process may not exist in the case of $E$. faecalis infection. Because gon-2 is known to be highly expressed in the intestine, where it is responsible for electrolyte homeostasis (Teramoto et al., 2005), it may function as an intestinal receptor for the changes elicited by $E$. faecalis colonization. Magnesium excretion, and perhaps additional electrolytes, requires the activity of $g t /-2$ in the excretory cell (Teramoto et al., 2010). Thus, it is possible that the role of gon-2 and gt/-2 in pathogen avoidance is related to sensation of an electrolyte perturbance caused by intestinal distention. These TRPM channels could also be mechano-nociceptors, as described in other animal models (MuellerTribbensee et al., 2015), that sense intestinal distention directly. Because the intestine is not directly innervated, any signal coming from it must be extra-synaptic, such as an intestinal neuropeptide (Lee and Mylonakis, 2017). All animals are under pressure to develop behaviors 
that allow them to flee potential pathogens (Sarabian et al., 2018). Future work will continue to elucidate the mechanisms underlying avoidance of pathogenic threats.

\section{MATERIALS AND METHODS}

\section{Bacterial strains}

The following bacterial strains were used: Enterococcus faecalis OG1RF, E. faecalis OG1RF $\Delta f s r B$, E. faecalis OG1RF-GFP, E. faecium E007, Escherichia coli OP50, E. coli OP50-GFP, E. coli HT115(DE3), Pseudomonas aeruginosa PA14, P. aeruginosa PA14-GFP, and Staphylococcus aureus NCTC8325. E. coli and P. aeruginosa bacterial strains were grown in Luria-Bertani (LB) broth at $37^{\circ} \mathrm{C}$ while the rest were grown in brain-heart infusion (BHI) broth at $37^{\circ} \mathrm{C}$. All sources are listed in the Key Resources table.

\section{C. elegans strains and growth conditions}

C. elegans hermaphrodites were maintained on $E$. coli at $20^{\circ} \mathrm{C}$ unless otherwise indicated. Bristol N2 was used as the wild-type control unless otherwise indicated. All other strains and their sources are listed in the Key Resources table.

\section{RNA interference (RNAi)}

RNAi was used to generate loss-of-function RNAi phenotypes by feeding nematodes $E$. coli strain HT115(DE3) expressing double-stranded RNA (dsRNA) homologous to a target gene (Fraser et al., 2000; Timmons and Fire, 1998). RNAi was carried out as described previously (Singh and Aballay, 2017). Briefly, E. coli with the appropriate vectors were grown in LB broth containing ampicillin $(100 \mu \mathrm{g} / \mathrm{mL})$ and tetracycline $(12.5 \mu \mathrm{g} / \mathrm{mL})$ at $37^{\circ} \mathrm{C}$ overnight and plated onto NGM plates containing $100 \mu \mathrm{g} / \mathrm{mL}$ ampicillin and $3 \mathrm{mM}$ isopropyl $\beta$-D-thiogalactoside (IPTG) (RNAi plates). RNAi-expressing bacteria were allowed to grow for two days at $20^{\circ} \mathrm{C}$. Gravid adults were transferred to RNAi-expressing bacterial lawns and allowed to lay eggs for 5 hr. The gravid adults were removed, and the eggs were allowed to develop at $20^{\circ} \mathrm{C}$ to young adults for subsequent assays. The RNAi clones were from the Ahringer RNAi library.

\section{Lawn avoidance assays}

Bacterial cultures were grown by inoculating individual bacterial colonies into $2 \mathrm{~mL}$ of either LB or $\mathrm{BHI}$ broth and growing them for $5-6 \mathrm{hr}$ on a shaker at $37^{\circ} \mathrm{C}$. Then, $20-\mu \mathrm{L}$ of the culture was plated onto the center of 3.5-cm-diameter BHI or standard slow-killing (SK) plates (modified NGM agar plates ( $0.35 \%$ instead of $0.25 \%$ peptone)) as indicated. The plates were then incubated overnight at $37^{\circ} \mathrm{C}$. The plates were cooled to room temperature for at least $30 \mathrm{~min}$ before seeding with animals. Synchronized young gravid adult hermaphroditic animals grown on E. coli OP50 were transferred outside the indicated bacterial lawns, and the numbers of animals on and off the lawns were counted at the specified times for each experiment. Three 


\section{5-cm-diameter plates were used per trial in every experiment. Occupancy index was} calculated as ( $\mathrm{N}_{\text {on }}$ lawn/ $\left./ \mathrm{N}_{\text {total }}\right)$. For first exiting events, individual animals were monitored and the time that the animal first left the lawn was recorded. For exploratory events entry and exiting events were recorded for 10 minutes at 50 minutes and 3 hours and 50 minutes after transfer.

\section{Avoidance assays at $8 \%$ oxygen}

Avoidance assays as described above were carried out in a hypoxia chamber. Briefly, after young gravid adult hermaphroditic animals were transferred to the avoidance plates, the plates were placed in the hypoxia chamber and the lids of the plates were removed. The chamber was purged with $8 \%$ oxygen (balanced with nitrogen) for $5 \mathrm{~min}$ at a flow rate of $25 \mathrm{~L} / \mathrm{min}$. The chamber was then sealed and assays were carried out. Control plates were incubated at ambient oxygen.

\section{Avoidance assays with bacterial RNA}

RNA from bacterial pellets was isolated and used for avoidance assays as previously described (Kaletsky et al., 2020). Briefly, bacteria for RNA collection were grown on SK or BHI plates overnight at $37^{\circ} \mathrm{C}$. Bacterial lawns were collected from the surface of the plates using $1 \mathrm{~mL}$ of M9 buffer and a cell scraper. The resulting suspension was transferred to a $15-\mathrm{mL}$ conical tube. PA14, OG1RF, or OP50 from 15 lawns was pooled in each tube and pelleted at 5,000g for 10 min at $4^{\circ} \mathrm{C}$. The supernatant was discarded and the pellet was resuspended in $1 \mathrm{~mL}$ of Trizol LS for every $100-\mu \mathrm{L}$ of bacterial pellet recovered. The pellet was resuspended by vortexing and subsequently frozen at $-80^{\circ} \mathrm{C}$ until RNA isolation. $240 \mu \mathrm{g}$ of total RNA was placed directly onto OP50 lawns and allowed to dry at room temperature before transferring worms over for avoidance assays.

\section{Aversive training}

3.5-cm-diameter training plates containing either E. coli OP50 on SK agar or E. faecalis OG1RF on $\mathrm{BHI}$ agar were made as described above for avoidance assays. For RNA training assays, SK plates containing $E$. coli OP50 were spotted with the appropriate isolated RNA. Young gravid adult hermaphroditic animals grown on E. coli OP50 were transferred to the training plates and allowed to roam for 4 hours for $E$. faecalis training or 24 hours for RNA training. They were then transferred to the appropriate assay plates.

\section{Two-choice preference assays}

Bacterial cultures were grown as indicated in the lawn avoidance assays above. Then, $20-\mu \mathrm{L}$ of each inoculum was plated on opposite sides of a 6 -cm-diameter $\mathrm{BHI}$ or SK plate and incubated overnight at $37^{\circ} \mathrm{C}$. The plates were cooled to room temperature for at least $30 \mathrm{~min}$ before 
seeding with animals. For the "Paralyzed" condition, $1 \mu \mathrm{L}$ of $1 \mathrm{M}$ sodium azide was spotted onto each bacterial lawn. Young gravid adult hermaphroditic animals grown on E. coli OP50 were transferred to the center of plates equidistant from both the lawns. The numbers of animals on both lawns were counted at the specified times for each experiment. Three 6-cm-diameter plates were used per trial in every experiment. The $E$. faecalis choice index (E. faecalis $\mathrm{Cl}$ ) was calculated as follows:

$$
\text { E. faecalis } \mathrm{CI}=\frac{[(\text { No. of worms on } E \text {.faecalis })-(\text { No. of worms on } E \text {.coli })]}{[(\text { No. of worms on } \text { E.faecalis })+(\text { No. of worms on } E \text {. coli })]}
$$

Choice indices to other bacteria were similarly calculated.

\section{Two-choice odor preference assays (lid choice assays)}

A modified version of the two-choice preference assays was carried out as previously described (Worthy et al., 2018) to assess odor preference alone. To do this, bacterial cultures were grown and plated as indicated in the two-choice preference assays above. Then, agar plugs with the bacterial lawns were cut from the plates and transferred to the lids of new 6 -cm-diameter $\mathrm{BHI}$ agar plates without any bacteria so that the bacteria on the agar plugs faced down towards the plate surface on opposite sides of the plate. $2 \mu \mathrm{L}$ of $1 \mathrm{M}$ sodium azide was spotted on the surface below each agar plug. Young gravid adult hermaphroditic animals grown on E. coli OP50 (naïve) or from E. faecalis OG1RF training plates were transferred to the center of plates equidistant from both agar plugs. The numbers of animals paralyzed under each agar plug were counted after one hour. Three 6-cm-diameter plates were used per trial in every experiment. The choice index was calculated as indicated above.

\section{Dry Drop Assay}

The dry drop assay was carried out as previously described (Tran et al., 2017). Using a capillary, a dry drop of either SDS (positive control), BHI or LB (negative control), or E. faecalis or $E$. faecium cultures was placed on a dry SK plate in front of a forward-moving animal. A response was counted if an animal initiated backward movement. Response Index = Number of Responses / Total Number of Drops.

\section{Imaging and quantification}

Fluorescence imaging was carried out as described previously (Singh and Aballay, 2017) with slight modifications. Briefly, the animals were anesthetized using an M9 salt solution containing $50 \mathrm{mM}$ sodium azide and mounted onto $2 \%$ agar pads. The animals were then visualized using a Leica M165 FC fluorescence stereomicroscope. For quantification of intestinal lumen bloating, brightfield images were acquired at each time point using the Leica LAS v4.6 software and the diameter of the intestinal lumen was measured using ImageJ software. For quantification of 
fluorescent immune reporters, fluorescent images were acquired using the Leica LAS v4.6 software in greyscale as presented, and the fluorescence intensity was measured and averaged across three points in the intestine of each animal at the indicated time points using ImageJ software.

\section{Statistical analysis}

The statistical analysis was performed with Prism 8 (GraphPad). For box-and-whisker plots, the center line depicts the median and the box range depicts the first and third quartile, while the whiskers depict the minimum and maximum data points. For all box plots, individual dots represent individual trials. For time course figures, the mean and standard deviation of 9 trials is depicted at each time point. All experiments were performed in triplicate on three separate days. Unpaired t-tests, One-way or two-way ANOVA with subsequent group comparisons were performed as indicated in the figure legends. In the figures, ns denotes not significant and asterisks $(*)$ denote statistical significance as follows: ${ }^{*}, \mathrm{p} \leq 0.05 ; * *, \mathrm{p} \leq 0.01 ; * * *, \mathrm{p} \leq 0.001 ; * * * *$, $\mathrm{p} \leq 0.0001$, as compared with the appropriate controls.

\section{ACKNOWLEDGEMENTS}

We thank Danielle A. Garsin (McGovern Medical School) for providing the E. faecalis OG1RF $\triangle f s r B$ and GFP strains. Some strains used in this study were provided by the Caenorhabditis Genetics Center (CGC), which is funded by the NIH Office of Research Infrastructure Programs (P400D01044). Images were created using Microsoft PowerPoint and BioRender.com.

\section{FIGURE LEGENDS}

Figure 1. E. faecalis elicits fast avoidance in C. elegans. (A) Schematic of avoidance assays (top) and representative photomicrographs (bottom) of $C$. elegans on lawns of $E$. coli OP50, $P$. aeruginosa PA14, or E. faecalis OG1RF at 0 hours (left) and 4 hours (right) on BHI media. Individual animals are outlined in red. Scale bar, $5 \mathrm{~mm}$. (B) Occupancy index of N2 animals after 4 hours of incubation on E. coli, P. aeruginosa, E. faecalis, E. faecium, or S. aureus. One-way ANOVA with subsequent comparison to $E$. coli as the control group was performed. Occupancy Index = (Number of animals on bacterial lawn) / (Total Number of animals). (C) Individual animals were tracked on lawns of $E$. faecalis and the time that they first left the lawn after transfer was recorded. Wild-type (WT, N2 Bristol) animals showed an average first leave time of 19.20 minutes while tax-2(p671) animals showed an average first leave time of 61.98 minutes. An unpaired t-test between the groups was performed. (D) N2 animals on standard avoidance assays plates with $E$. faecalis were observed for 10 minutes during two different time windows (0-1 hours and 3-4 hours) and the number of times the animals left and entered the bacteria lawns was counted. One-way ANOVA with subsequent comparisons between all groups was performed. (E) Occupancy index of N2 animals after 24-hour incubation on P. aeruginosa PA14 
lawns or E. coli OP50 lawns supplemented with isolated total RNA from E. coli (E.c. RNA), P. aeruginosa (P.a. RNA), or E. faecalis (E.f. RNA). One-way ANOVA with subsequent comparison to $E$. coli RNA as the control group was performed.

Figure 2: Anterior intestinal distention elicits avoidance of E. faecalis. (A) Representative photomicrographs of animals at 4 hours on BHI media with E. coli (left), P. aeruginosa (middle), or E. faecalis (right). Red arrows point to the borders of the anterior intestine while blue arrows point the borders of the medial intestine. (B) Quantification of the anterior (top) and medial (bottom) intestinal lumen diameter of animals on E. coli, P. aeruginosa, or E. faecalis at 1, 2, and 4 hours. Two-way ANOVA was performed with comparison to the $E$. coli group at each timepoint. (C) Representative photomicrographs with fluorescent and brightfield images merged of E. coli, $P$. aeruginosa, and $E$. faecalis expressing GFP in the intestinal lumen of animals at 4 hours. (D) Occupancy index on E. faecalis at 4 hours of WT animals compared to either RNAi-mediated knockdown of nol-6 or a loss-of-function mutation in nol-6. One-way ANOVA was performed with subsequent comparison to the WT group as the control. (E) A representative photomicrograph of a nol-6(ac1) animal after exposure to $E$. faecalis for 4 hours. Red arrows point to the borders of the anterior intestine and blue arrows point to the borders of the medial intestine. (F) Quantification of anterior and medial intestinal lumen diameter of nol-6(ac1) animals at 0 and 4 hours on E. faecalis. Two-way ANOVA was performed with comparison to the 0 -hour groups for both anterior and medial quantifications.

Figure 3. Avoidance of $E$. faecalis is independent of virulence. (A) Occupancy index of N2 animals on virulent $E$. faecalis OG1RF and avirulent $E$. faecalis OG1RF $\Delta f s r B$ at 4 hours. An unpaired t-test was performed. (B) Representative fluorescent micrographs of clec-60p::GFP (top) and ilys-3p::GFP (bottom) animals at 0, 4, and 24 hours on E. faecalis. Scale bar, $200 \mu \mathrm{m}$. (C) Quantification of clec-60::GFP at 0, 4, and 24 hours on E. faecalis. One-way ANOVA was performed with comparisons to the 0-hour group as the control. (D) Quantification of ilys3p::GFP at 0, 4, and 24 hours on E. faecalis. One-way ANOVA was performed with comparisons to the 0 -hour group as the control.

Figure 4. NPR-1 and TAX-2/4 pathways regulate avoidance of E. faecalis and P. aeruginosa. (A) Occupancy index of WT animals compared to npr-1(ad609) and npr-1(ok1447) after 4 hours on E. faecalis. One-way ANOVA with subsequent comparison to WT animals as the control group was performed. (B) Occupancy index of WT, npr-1(ad609) and npr-1(ok1447) on lawns of $E$. faecalis in atmospheric oxygen or a chamber containing 8\% oxygen. Two-way ANOVA with subsequent comparison to WT animals of each respective oxygen condition was performed. (C) Occupancy index of WT, npr-1(ad609), and npr-1(ok1447) in an 8\% oxygen chamber at 1, 2, 3, and 4 hours, and 1 hour after removal from the chamber (vertical dashed line). Two-way ANOVA with subsequent comparison at each time point to WT animals was performed. (D) Occupancy index for WT and double loss-of-function mutants for gcy-35, tax-2, or tax-4 and 
npr-1 on E. faecalis for 4 hours and P. aeruginosa for 24 hours. Two-way ANOVA with subsequent comparisons to WT animals for each bacterium were performed. (E) Diagram of the neurons affected by tax-2(p694) and tax-4(p678). The latter allele covers all tax-2 expressing sensory neurons, while the former covers a subset. (F) Occupancy index for WT and single lossof-function mutants for tax-2 or tax-4 on E. faecalis for 4 hours and $P$. aeruginosa for 24 hours. Two-way ANOVA as in with subsequent comparisons to WT animals for each bacterium were performed.

Figure 5. ASE, AWB, and AWC neurons mediate avoidance of E. faecalis. (A) Occupancy index on E. faecalis at 4 hours for WT, tax-2(p694) and animals with tax-2 expression in ASE neurons (flp6p::tax-2) or AQR, PQR, or URX neurons (gcy-32p::tax-2) in the tax-2(p694) background. Oneway ANOVA with subsequent comparison to wild-type (WT) animals was performed. (B) Occupancy index of WT animals and animals with ablated neurons on E. faecalis at 4 hours and $P$. aeruginosa at 24 hours. Ablation of sensory neurons either by mutation (che-1 $(p 680)=$ ASE()) or caspase expression (sra-6p::mCasp-1 = ASH(-); gpa-4p::TU\#813 + gcy-27p::TU\#814 = ASI(-); str-1p::mCasp-1 = AWB(-); ceh-36p::TU\#813 + ceh-36p::TU\#814 = AWC(-)). Two-way ANOVA with subsequent comparisons to WT groups for each respective bacterium were performed. (C) Occupancy index at 1 hour for WT and ASI(-) animals on $P$. aeruginosa lawns. Animals were trained on E. coli OP50 lawns supplemented with either $E$. coli or $P$. aeruginosa RNA for 24 hours Two-way ANOVA with subsequent comparison with WT control groups was performed. (D) Choice index after 1 hour for WT and ASI(-) animals choosing between $E$. coli and $P$. aeruginosa lawns. Two-way ANOVA with subsequent comparison with WT control groups was performed. Choice Index $=$ (Number of animals on $E$. coli - Number of animals on $P$. aeruginosa) / (Number of animals on E. coli + Number of animals on $P$. aeruginosa). (E) Model for avoidance of $P$. aeruginosa (top) and $E$. faecalis (bottom) Avoidance of $P$. aeruginosa depends on both an ASI neuron-mediated bacterial sRNA pathway along with intestine-wide distention. The latter requires the NPR-1-dependent hyperoxia avoidance pathway along with AWB and AWC olfactory neurons. Avoidance of $E$. faecalis also depends on intestinal distention, though this is confined to the anterior intestine. This anterior intestinal distention-induced avoidance also requires NPR-1-dependent hyperoxia avoidance and AWB and AWC olfactory neurons, but also requires ASE chemosensory neurons.

Figure 6. AWB and AWC neurons are necessary for aversive olfactory learning following ingestion of $E$. faecalis. (A) Schematic of the choice assay (top) and quantification (bottom) of choice index for $\mathrm{N} 2$ animals choosing between $E$. coli and E. faecalis lawns and either free to move for 1 hour (Free to move) or paralyzed upon arrival at a bacterial lawn (Paralyzed). An unpaired t-test between the groups was performed. (B) Schematic of the lid choice assay (top) and quantification of naïve and trained choice index (bottom, left) and learning index (bottom, right) for WT, tax-4(p678), $\operatorname{tax}-2(p 671), \operatorname{tax}-2(p 694), A W B(-)$, and AWC(-) animals. To train 
animals, young adult animals were placed on E. faecalis lawns for 4 hours before the choice assay was performed. For choice index, two-way ANOVA with subsequent comparisons to the naïve and trained WT groups as controls were performed. For learning index, one-way ANOVA with comparison to the WT group as control was performed. Learning index = (Naïve choice index) - (Trained choice index).

Figure 7. The TRPM channels GON-2 and GTL-2 are required for distention-induced pathogen avoidance. (A) Occupancy index for wild-type, gtl-2(n2618), gon-2(q362), gon-2(q388), and glp1(e2141) animals on E. faecalis at 4 hours or $P$. aeruginosa at 24 hours. Animals were grown to the young adult stage at $15^{\circ} \mathrm{C}$. Two-way ANOVA with comparisons to the respective WT group for each bacterium were performed. (B) Occupancy index for wild-type, gt/-2(n2618), gon2(q362), gon-2(q388), and glp-1(e2141) animals on E. faecalis at 4 hours or P. aeruginosa at 24 hours. Animals were grown to the young adult stage at $25^{\circ} \mathrm{C}$. Two-way ANOVA with comparisons to the respective WT group for each bacterium were performed. (C) Occupancy index for WT, gon-2(q388) and gtl-2(n2618) animals with either RNAi-mediated knockdown of aex-5 or an empty vector (EV) control on the E. coli HT115 lawns these animals were raised on. Two-way ANOVA with subsequent comparison between all groups was performed. (D) Occupancy index for WT, gon-2(q388) and gt/-2(n2618) animals with either RNAi-mediated knockdown of aex-5 or an EV control on E. faecalis lawns at 4 hours. Two-way ANOVA with subsequent comparison between all groups was performed. (E) Model for avoidance of $P$. aeruginosa (top) and E. faecalis (bottom), as in Figure $\mathbf{5 E}$, with the addition of a germline role in avoidance of $P$. aeruginosa, GON-2 and GTL-2 regulation of avoidance of $E$. faecalis, and the contribution of odor sensing pathways to avoidance for avoidance of both bacteria.

Figure 1 - Supplement 1. Avoidance of Gram-positive pathogens. (A) Occupancy index time course of N2 animals on either E. faecalis, E. faecium, or S. aureus. (B) Response index of N2 animals to dry drops of E. coli, E. faecalis, E. faecium, BHI, LB, or 0.6mM SDS buffer. Response Index $=$ Number of Responses / Total Number of Drops.

Figure 2 - Supplement 2. E. faecalis but not $P$. aeruginosa causes anterior intestinal distention in nol-6 animals. (A) Quantification of anterior and medial intestinal lumen diameter of nol6(ac1) animals after 0 and 24 hours on $P$. aeruginosa. (B) Occupancy index of WT and nol-6(ac1) animals on $P$. aeruginosa at 24 hours. An unpaired t-test was performed. (C) Quantification of anterior intestinal lumen diameter of nol-6 RNAi animals at 0 and 4 hours on E. faecalis. An unpaired t-test was performed. (D) Quantification of medial intestinal lumen diameter of nol-6 RNAi animals at 0 and 4 hours on E. faecalis. An unpaired t-test was performed.

Figure 4 -Supplement 1. The TRPV subunits OCR-2 and OSM-9 slightly speed up avoidance of $E$. faecalis. (A) Occupancy index of WT animals compared to ocr-2(ak47) and osm-9(yz6) animals on E. faecalis at 4 hours. One-way ANOVA with subsequent comparison to WT animals was 
performed. (B) Occupancy index time course for WT animals compared to ocr-2(ak47) and osm9(yz6) on E. faecalis. Two-way ANOVA with comparison to WT animals at each time point was performed.

Figure 7 - Supplement 1. Screening TRP channels for effect on avoidance of E. faecalis. Occupancy index of WT animals on E. faecalis at 4 hours compared to 5 different TRP channel mutants: gon-2(q362), gon-2(q388), gtl-2(n2618), and gt/-2(tm1643). One-way ANOVA with subsequent comparison to WT animals was performed.

\section{ADDITIONAL INFORMATION}

\section{Funding}

This work was supported by a National Institute of General Medical Sciences grant (GM070907) and a National Institute of Allergy and Infectious Diseases grant (Al11791) to Alejandro Aballay.

\section{Author contributions}

Adam Filipowicz, Conceptualization, Data curation, Formal analysis, Investigation, Methodology, Writing - original draft, Writing - review and editing; Alejandro Aballay, Conceptualization, Data curation, Formal analysis, Funding acquisition, Writing - original draft, Project administration, Writing - review and editing

\section{REFERENCES}

Anderson, A., and McMullan, R. (2018). Neuronal and non-neuronal signals regulate Caenorhabditis elegans avoidance of contaminated food. Philos. Trans. R. Soc. B Biol. Sci. 373.

Austin, J., and Kimble, J. (1987). glp-1 is required in the germ line for regulation of the decision between mitosis and meiosis in C. elegans. Cell 51, 589-599.

de Bono, M., and Bargmann, C.I. (1998). Natural variation in a neuropeptide Y receptor homolog modifies social behavior and food response in C. elegans. Cell 94, 679-689.

de Bono, M., Tobin, D.M., Davis, M.W., Avery, L., and Bargmann, C.I. (2002). Social feeding in Caenorhabditis elegans is induced by neurons that detect aversive stimuli. Nature 419, 899903.

Bretscher, A.J., Kodama-Namba, E., Busch, K.E., Murphy, R.J., Soltesz, Z., Laurent, P., and de Bono, M. (2011). Temperature, Oxygen, and Salt-Sensing Neurons in C. elegans Are Carbon Dioxide Sensors that Control Avoidance Behavior. Neuron 69, 1099-1113.

Cao, X., Kajino-Sakamoto, R., Doss, A., and Aballay, A. (2017). Distinct Roles of Sensory Neurons in Mediating Pathogen Avoidance and Neuropeptide-Dependent Immune Regulation. Cell Rep. 21, 1442-1451. 
Chalasani, S.H., Chronis, N., Tsunozaki, M., Gray, J.M., Ramot, D., Goodman, M.B., and Bargmann, C.I. (2007). Dissecting a circuit for olfactory behaviour in Caenorhabditis elegans. Nature 450, 63-70.

Chalasani, S.H., Kato, S., Albrecht, D.R., Nakagawa, T., Abbott, L.F., and Bargmann, C.I. (2010). Neuropeptide feedback modifies odor-evoked dynamics in Caenorhabditis elegans olfactory neurons. Nat. Neurosci. 13, 615-621.

Chang, A.J., Chronis, N., Karow, D.S., Marletta, M.A., and Bargmann, C.I. (2006). A Distributed Chemosensory Circuit for Oxygen Preference in C. elegans. PLOS Biol. 4, e274.

Chen, Z., Hendricks, M., Cornils, A., Maier, W., Alcedo, J., and Zhang, Y. (2013). Two Insulin-like Peptides Antagonistically Regulate Aversive Olfactory Learning in C. elegans. Neuron 77, 572585.

Cheung, B.H.H., Arellano-Carbajal, F., Rybicki, I., and Bono, M. de (2004). Soluble Guanylate Cyclases Act in Neurons Exposed to the Body Fluid to Promote C. elegans Aggregation Behavior. Curr. Biol. 14, 1105-1111.

Coates, J.C., and de Bono, M. (2002). Antagonistic pathways in neurons exposed to body fluid regulate social feeding in Caenorhabditis elegans. Nature 419, 925-929.

Coburn, C.M., and Bargmann, C.I. (1996). A Putative Cyclic Nucleotide-Gated Channel Is Required for Sensory Development and Function in C. elegans. Neuron 17, 695-706.

Crittenden, S.L., Troemel, E.R., Evans, T.C., and Kimble, J. (1994). GLP-1 is localized to the mitotic region of the C. elegans germ line. Dev. Camb. Engl. 120, 2901-2911.

Engelmann, I., Griffon, A., Tichit, L., Montañana-Sanchis, F., Wang, G., Reinke, V., Waterston, R.H., Hillier, L.W., and Ewbank, J.J. (2011). A Comprehensive Analysis of Gene Expression Changes Provoked by Bacterial and Fungal Infection in C. elegans. PLoS ONE 6.

Fadda, M., Fruyt, N.D., Borghgraef, C., Watteyne, J., Peymen, K., Vandewyer, E., Galindo, F.J.N., Kieswetter, A., Mirabeau, O., Chew, Y.L., et al. (2020). NPY/NPF-Related Neuropeptide FLP-34 Signals from Serotonergic Neurons to Modulate Aversive Olfactory Learning in Caenorhabditis elegans. J. Neurosci. 40, 6018-6034.

Flavell, S.W., Pokala, N., Macosko, E.Z., Albrecht, D.R., Larsch, J., and Bargmann, C.I. (2013). Serotonin and the Neuropeptide PDF Initiate and Extend Opposing Behavioral States in $C$. elegans. Cell 154, 1023-1035.

Fraser, A.G., Kamath, R.S., Zipperlen, P., Martinez-Campos, M., Sohrmann, M., and Ahringer, J. (2000). Functional genomic analysis of $C$. elegans chromosome I by systematic RNA interference. Nature 408, 325-330. 
Fuhrman, L.E., Goel, A.K., Smith, J., Shianna, K.V., and Aballay, A. (2009). Nucleolar Proteins Suppress Caenorhabditis elegans Innate Immunity by Inhibiting p53/CEP-1. PLoS Genet. 5.

Garsin, D.A., Sifri, C.D., Mylonakis, E., Qin, X., Singh, K.V., Murray, B.E., Calderwood, S.B., and Ausubel, F.M. (2001). A simple model host for identifying Gram-positive virulence factors. Proc. Natl. Acad. Sci. 98, 10892-10897.

Gravato-Nobre, M.J., Vaz, F., Filipe, S., Chalmers, R., and Hodgkin, J. (2016). The Invertebrate Lysozyme Effector ILYS-3 Is Systemically Activated in Response to Danger Signals and Confers Antimicrobial Protection in C. elegans. PLoS Pathog. 12.

Gray, J.M., Karow, D.S., Lu, H., Chang, A.J., Chang, J.S., Ellis, R.E., Marletta, M.A., and Bargmann, C.I. (2004). Oxygen sensation and social feeding mediated by a $C$. elegans guanylate cyclase homologue. Nature 430, 317-322.

Ha, H., Hendricks, M., Shen, Y., Gabel, C.V., Fang-Yen, C., Qin, Y., Colón-Ramos, D., Shen, K., Samuel, A.D.T., and Zhang, Y. (2010). Functional organization of a neural network for aversive olfactory learning in Caenorhabditis elegans. Neuron 68, 1173-1186.

Harris, G., Shen, Y., Ha, H., Donato, A., Wallis, S., Zhang, X., and Zhang, Y. (2014). Dissecting the Signaling Mechanisms Underlying Recognition and Preference of Food Odors. J. Neurosci. 34, 9389-9403.

Holzer, P. (2011). TRP channels in the digestive system. Curr. Pharm. Biotechnol. 12, 24-34.

Irazoqui, J.E., Troemel, E.R., Feinbaum, R.L., Luhachack, L.G., Cezairliyan, B.O., and Ausubel, F.M. (2010). Distinct Pathogenesis and Host Responses during Infection of $C$. elegans by $P$. aeruginosa and S. aureus. PLoS Pathog. 6.

Kaletsky, R., Moore, R.S., Vrla, G.D., Parsons, L.R., Gitai, Z., and Murphy, C.T. (2020). C. elegans interprets bacterial non-coding RNAs to learn pathogenic avoidance. Nature 1-7.

Kaplan, J.M., and Horvitz, H.R. (1993). A dual mechanosensory and chemosensory neuron in Caenorhabditis elegans. Proc. Natl. Acad. Sci. U. S. A. 90, 2227-2231.

Kavaliers, M., Ossenkopp, K.-P., and Choleris, E. (2019). Social neuroscience of disgust. Genes Brain Behav. 18, e12508.

Kindt, K.S., Viswanath, V., Macpherson, L., Quast, K., Hu, H., Patapoutian, A., and Schafer, W.R. (2007). Caenorhabditis elegans TRPA-1 functions in mechanosensation. Nat. Neurosci. 10, 568577.

Kiyama, Y., Miyahara, K., and Ohshima, Y. (2012). Active uptake of artificial particles in the nematode Caenorhabditis elegans. J. Exp. Biol. 215, 1178-1183. 
Kumar, S., Egan, B.M., Kocsisova, Z., Schneider, D.L., Murphy, J.T., Diwan, A., and Kornfeld, K. (2019). Lifespan Extension in C. elegans Caused by Bacterial Colonization of the Intestine and Subsequent Activation of an Innate Immune Response. Dev. Cell 49, 100-117.e6.

Lee, K., and Mylonakis, E. (2017). An Intestine-Derived Neuropeptide Controls Avoidance Behavior in Caenorhabditis elegans. Cell Rep. 20, 2501-2512.

Leinwand, S.G., and Chalasani, S.H. (2013). Neuropeptide signaling remodels chemosensory circuit composition in Caenorhabditis elegans. Nat. Neurosci. 16, 1461-1467.

Leinwand, S.G., Yang, C.J., Bazopoulou, D., Chronis, N., Srinivasan, J., and Chalasani, S.H. (2015). Circuit mechanisms encoding odors and driving aging-associated behavioral declines in Caenorhabditis elegans. ELife 4, e10181.

Medzhitov, R., Schneider, D.S., and Soares, M.P. (2012). Disease tolerance as a defense strategy. Science 335, 936-941.

Meisel, J.D., and Kim, D.H. (2014). Behavioral avoidance of pathogenic bacteria by Caenorhabditis elegans. Trends Immunol. 35, 465-470.

Meisel, J.D., Panda, O., Mahanti, P., Schroeder, F.C., and Kim, D.H. (2014). Chemosensation of Bacterial Secondary Metabolites Modulates Neuroendocrine Signaling and Behavior of $C$. elegans. Cell 159, 267-280.

Metaxakis, A., Petratou, D., and Tavernarakis, N. (2018). Multimodal sensory processing in Caenorhabditis elegans. Open Biol. 8.

Milward, K., Busch, K.E., Murphy, R.J., Bono, M. de, and Olofsson, B. (2011). Neuronal and molecular substrates for optimal foraging in Caenorhabditis elegans. Proc. Natl. Acad. Sci. 108, 20672-20677.

Moore, R.S., Kaletsky, R., and Murphy, C.T. (2019). Piwi/PRG-1 Argonaute and TGF- $\beta$ Mediate Transgenerational Learned Pathogenic Avoidance. Cell 177, 1827-1841.e12.

Mueller-Tribbensee, S.M., Karna, M., Khalil, M., Neurath, M.F., Reeh, P.W., and Engel, M.A. (2015). Differential Contribution of TRPA1, TRPV4 and TRPM8 to Colonic Nociception in Mice. PLOS ONE 10.

Mylonakis, E., Engelbert, M., Qin, X., Sifri, C.D., Murray, B.E., Ausubel, F.M., Gilmore, M.S., and Calderwood, S.B. (2002). The Enterococcus faecalis fsrB Gene, a Key Component of the fsr Quorum-Sensing System, Is Associated with Virulence in the Rabbit Endophthalmitis Model. Infect. Immun. 70, 4678-4681.

Olofsson, B. (2014). The olfactory neuron AWC promotes avoidance of normally palatable food following chronic dietary restriction. J. Exp. Biol. 217, 1790-1798. 
Pradel, E., Zhang, Y., Pujol, N., Matsuyama, T., Bargmann, C.I., and Ewbank, J.J. (2007). Detection and avoidance of a natural product from the pathogenic bacterium Serratia marcescens by Caenorhabditis elegans. Proc. Natl. Acad. Sci. U. S. A. 104, 2295-2300.

Reddy, K.C., Andersen, E.C., Kruglyak, L., and Kim, D.H. (2009). A Polymorphism in npr-1 Is a Behavioral Determinant of Pathogen Susceptibility in C. elegans. Science 323, 382-384.

Reddy, K.C., Hunter, R.C., Bhatla, N., Newman, D.K., and Kim, D.H. (2011). Caenorhabditis elegans NPR-1-mediated behaviors are suppressed in the presence of mucoid bacteria. Proc. Natl. Acad. Sci. U. S. A. 108, 12887-12892.

Rogers, C., Reale, V., Kim, K., Chatwin, H., Li, C., Evans, P., and de Bono, M. (2003). Inhibition of Caenorhabditis elegans social feeding by FMRFamide-related peptide activation of NPR-1. Nat. Neurosci. 6, 1178-1185.

Sarabian, C., Curtis, V., and McMullan, R. (2018). Evolution of pathogen and parasite avoidance behaviourst. Philos. Trans. R. Soc. B Biol. Sci. 373.

Shtonda, B.B., and Avery, L. (2006). Dietary choice behavior in Caenorhabditis elegans. J. Exp. Biol. 209, 89-102.

Sifri, C.D., Mylonakis, E., Singh, K.V., Qin, X., Garsin, D.A., Murray, B.E., Ausubel, F.M., and Calderwood, S.B. (2002). Virulence effect of Enterococcus faecalis protease genes and the quorum-sensing locus fsr in Caenorhabditis elegans and mice. Infect. Immun. 70, 5647-5650.

Singh, J., and Aballay, A. (2017). Endoplasmic Reticulum Stress Caused by Lipoprotein Accumulation Suppresses Immunity against Bacterial Pathogens and Contributes to Immunosenescence. MBio 8.

Singh, J., and Aballay, A. (2019a). Intestinal infection regulates behavior and learning via neuroendocrine signaling. ELife 8, e50033.

Singh, J., and Aballay, A. (2019b). Microbial Colonization Activates an Immune Fight-and-Flight Response via Neuroendocrine Signaling. Dev. Cell 49, 89-99.e4.

Singh, J., and Aballay, A. (2020). Neural control of behavioral and molecular defenses in $C$. elegans. Curr. Opin. Neurobiol. 62, 34-40.

Soldano, A., Alpizar, Y.A., Boonen, B., Franco, L., López-Requena, A., Liu, G., Mora, N., Yaksi, E., Voets, T., Vennekens, R., et al (2016). Gustatory-mediated avoidance of bacterial lipopolysaccharides via TRPA1 activation in Drosophila. ELife 5.

Stensmyr, M.C., Dweck, H.K.M., Farhan, A., Ibba, I., Strutz, A., Mukunda, L., Linz, J., Grabe, V., Steck, K., Lavista-Llanos, S., et al. (2012). A Conserved Dedicated Olfactory Circuit for Detecting Harmful Microbes in Drosophila. Cell 151, 1345-1357. 
Styer, K.L., Singh, V., Macosko, E., Steele, S.E., Bargmann, C.I., and Aballay, A. (2008). Innate Immunity in Caenorhabditis elegans Is Regulated by Neurons Expressing NPR-1/GPCR. Science $322,460-464$.

Summers, P.J., Layne, R.M., Ortega, A.C., Harris, G.P., Bamber, B.A., and Komuniecki, R.W. (2015). Multiple Sensory Inputs Are Extensively Integrated to Modulate Nociception in $C$. elegans. J. Neurosci. 35, 10331-10342.

Sun, A.Y., and Lambie, E.J. (1997). Gon-2, a Gene Required for Gonadogenesis in Caenorhabditis elegans. Genetics 147, 1077-1089.

TeKippe, M., and Aballay, A. (2010). C. elegans Germline-Deficient Mutants Respond to Pathogen Infection Using Shared and Distinct Mechanisms. PLoS ONE 5.

Teramoto, T., Lambie, E.J., and Iwasaki, K. (2005). Differential regulation of TRPM channels governs electrolyte homeostasis in the C. elegans intestine. Cell Metab. 1, 343-354.

Teramoto, T., Sternick, L.A., Kage-Nakadai, E., Sajjadi, S., Siembida, J., Mitani, S., Iwasaki, K., and Lambie, E.J. (2010). Magnesium Excretion in C. elegans Requires the Activity of the GTL-2 TRPM Channel. PLOS ONE 5, e9589.

Timmons, L., and Fire, A. (1998). Specific interference by ingested dsRNA. Nature 395, 854-854.

Tran, A., Tang, A., O'Loughlin, C.T., Balistreri, A., Chang, E., Coto Villa, D., Li, J., Varshney, A., Jimenez, V., Pyle, J., et al. (2017). C. elegans avoids toxin-producing Streptomyces using a seven transmembrane domain chemosensory receptor. ELife 6, e23770.

Wani, K.A., Goswamy, D., and Irazoqui, J.E. (2020). Nervous system control of intestinal host defense in C. elegans. Curr. Opin. Neurobiol. 62, 1-9.

Wilson, M.A., Iser, W.B., Son, T.G., Logie, A., Cabral-Costa, J.V., Mattson, M.P., and Camandola, S. (2017). skn-1 is required for interneuron sensory integration and foraging behavior in Caenorhabditis elegans. PLOS ONE 12.

Worthy, S.E., Rojas, G.L., Taylor, C.J., and Glater, E.E. (2018). Identification of Odor Blend Used by Caenorhabditis elegans for Pathogen Recognition. Chem. Senses 43, 169-180.

Xiao, R., and Xu, X.Z.S. (2011). C. elegans TRP channels. Adv. Exp. Med. Biol. 704, 323-339.

Xiao, R., Zhang, B., Dong, Y., Gong, J., Xu, T., Liu, J., and Xu, X.Z.S. (2013). A Genetic Program Promotes $C$. elegans Longevity at Cold Temperatures via a Thermosensitive TRP Channel. Cell $152,806-817$.

Yoshida, K., Hirotsu, T., Tagawa, T., Oda, S., Wakabayashi, T., lino, Y., and Ishihara, T. (2012). Odour concentration-dependent olfactory preference change in C. elegans. Nat. Commun. 3, 739. 
bioRxiv preprint doi: https://doi.org/10.1101/2020.12.18.423492; this version posted December 19,2020 . The copyright holder for this preprint (which was not certified by peer review) is the author/funder, who has granted bioRxiv a license to display the preprint in perpetuity. It is made available under aCC-BY-NC-ND 4.0 International license.

Yuen, G.J., and Ausubel, F.M. (2018). Both live and dead Enterococci activate Caenorhabditis elegans host defense via immune and stress pathways. Virulence 9, 683-699.

Zhang, Y., Lu, H., and Bargmann, C.I. (2005). Pathogenic bacteria induce aversive olfactory learning in Caenorhabditis elegans. Nature 438, 179-184. 

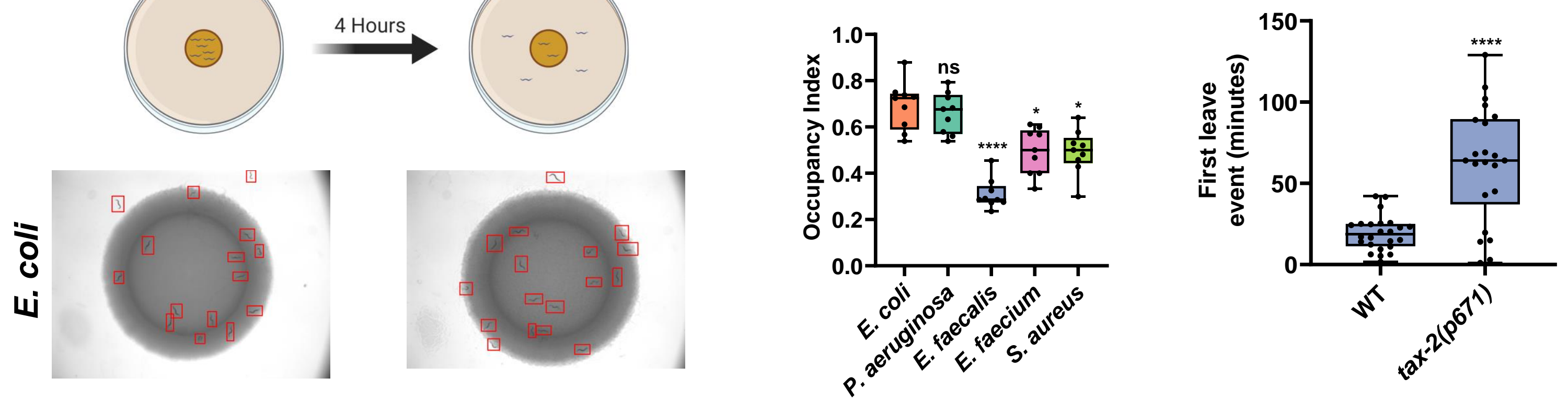

0
5
0
$\frac{5}{0}$
$\frac{5}{6}$
$\frac{1}{0}$
0

D

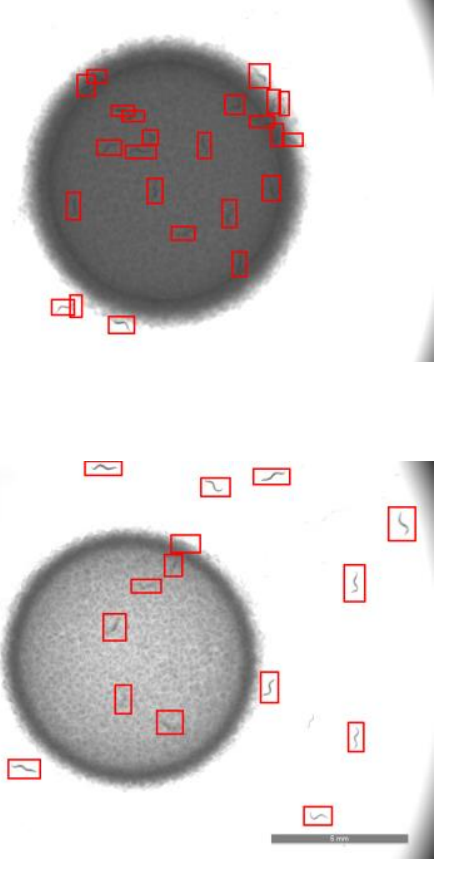

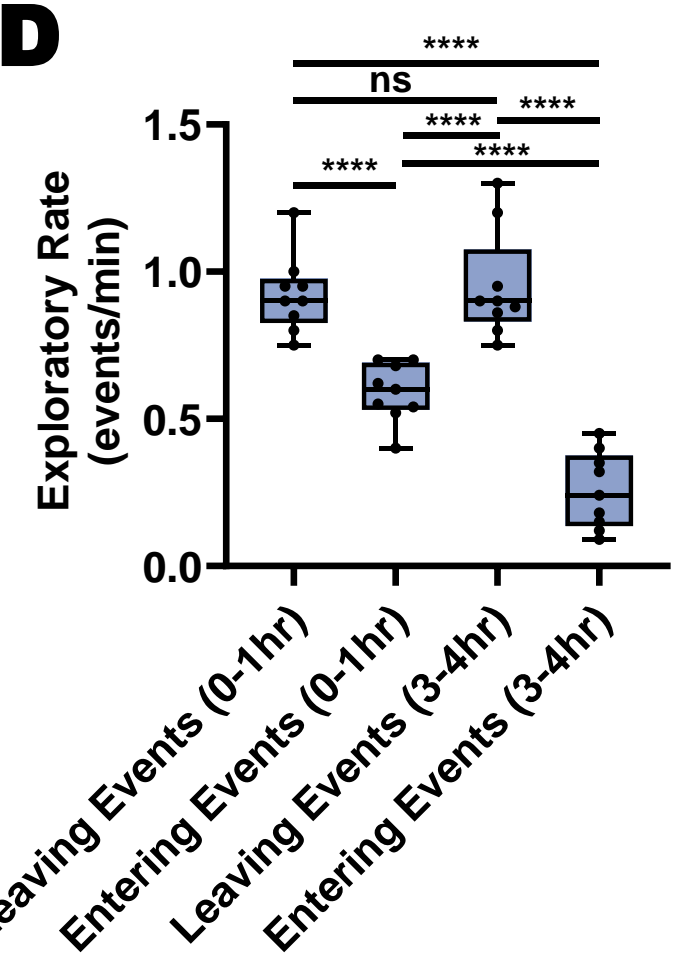

E

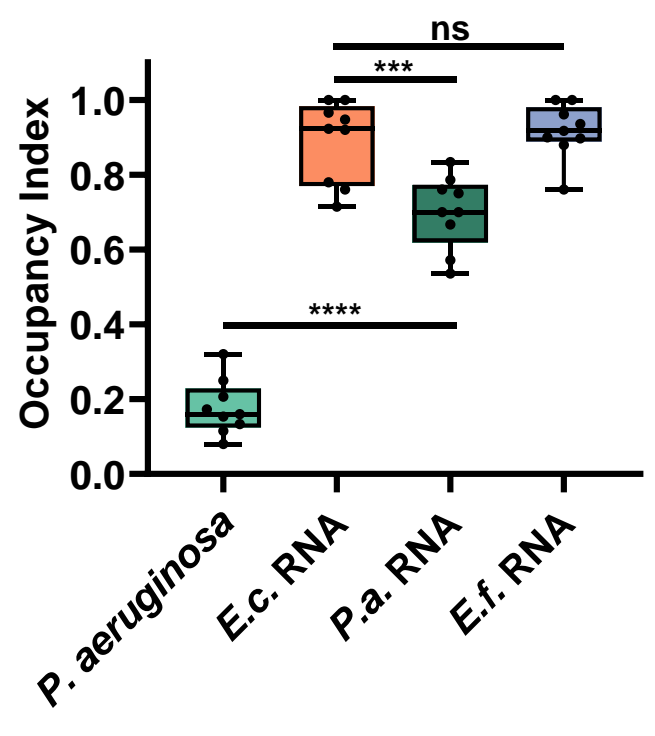

Figure 1 

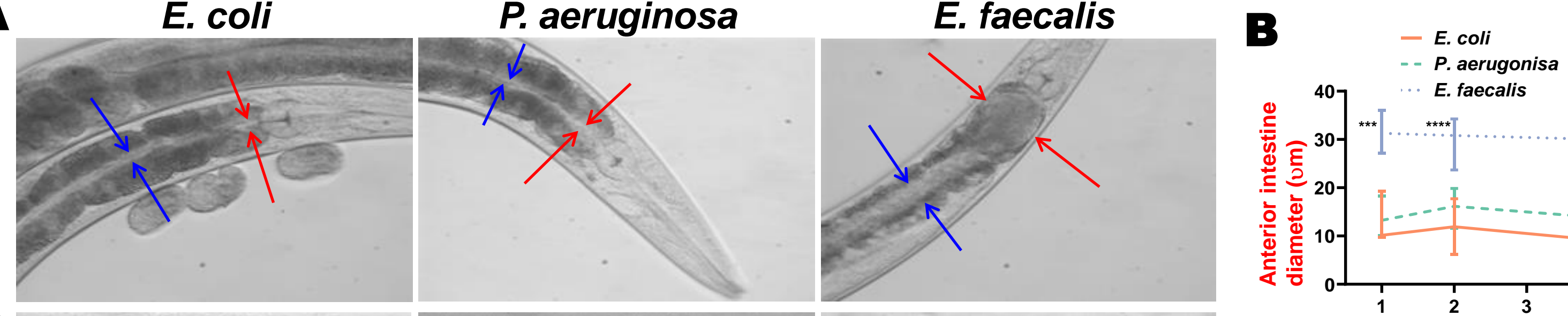

C

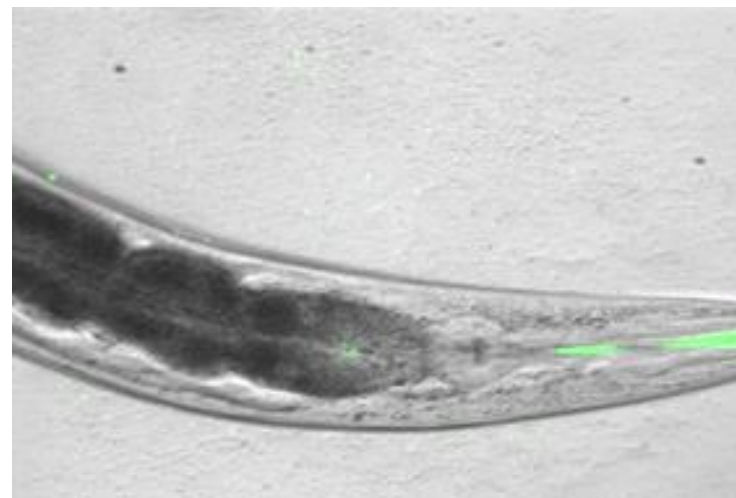

D

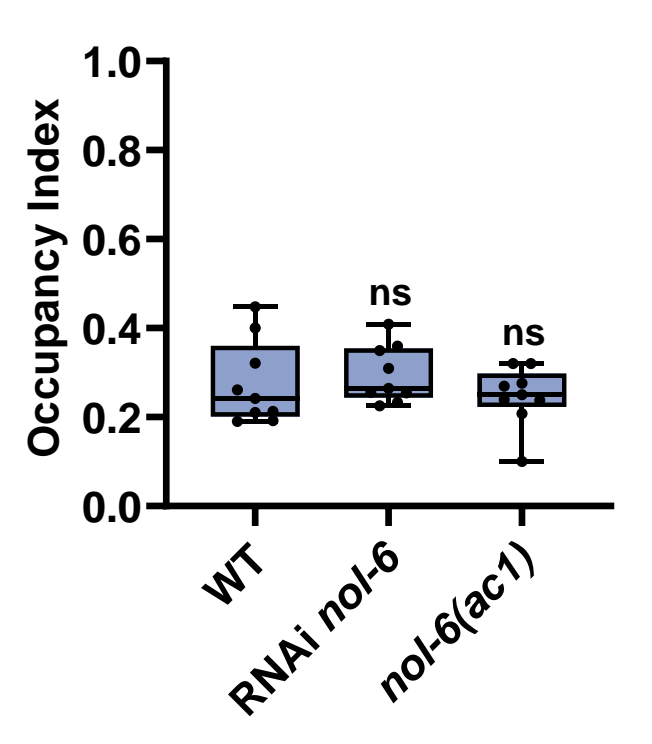

E

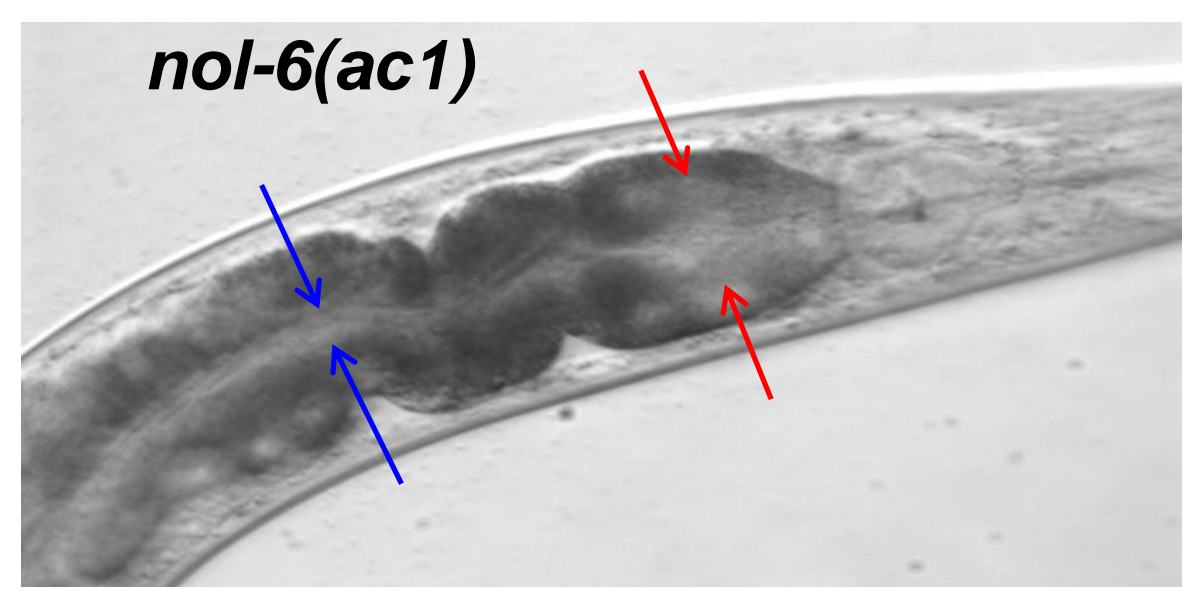

F

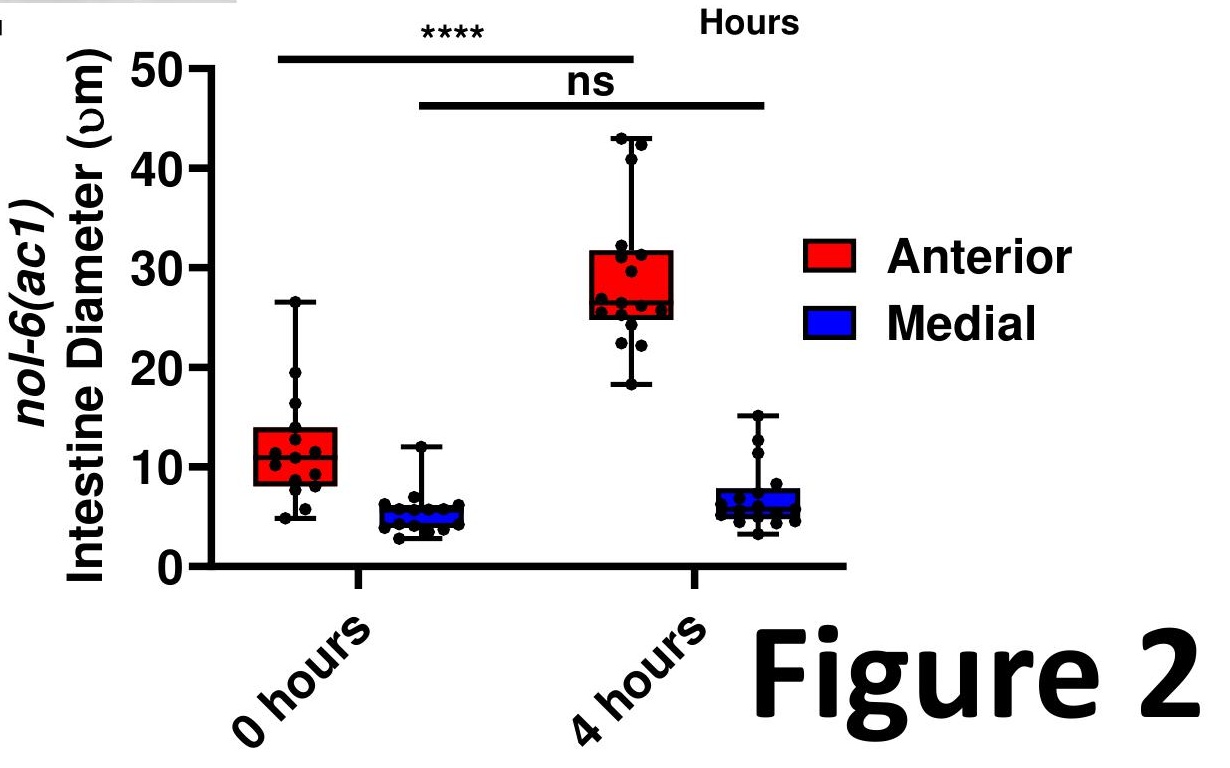



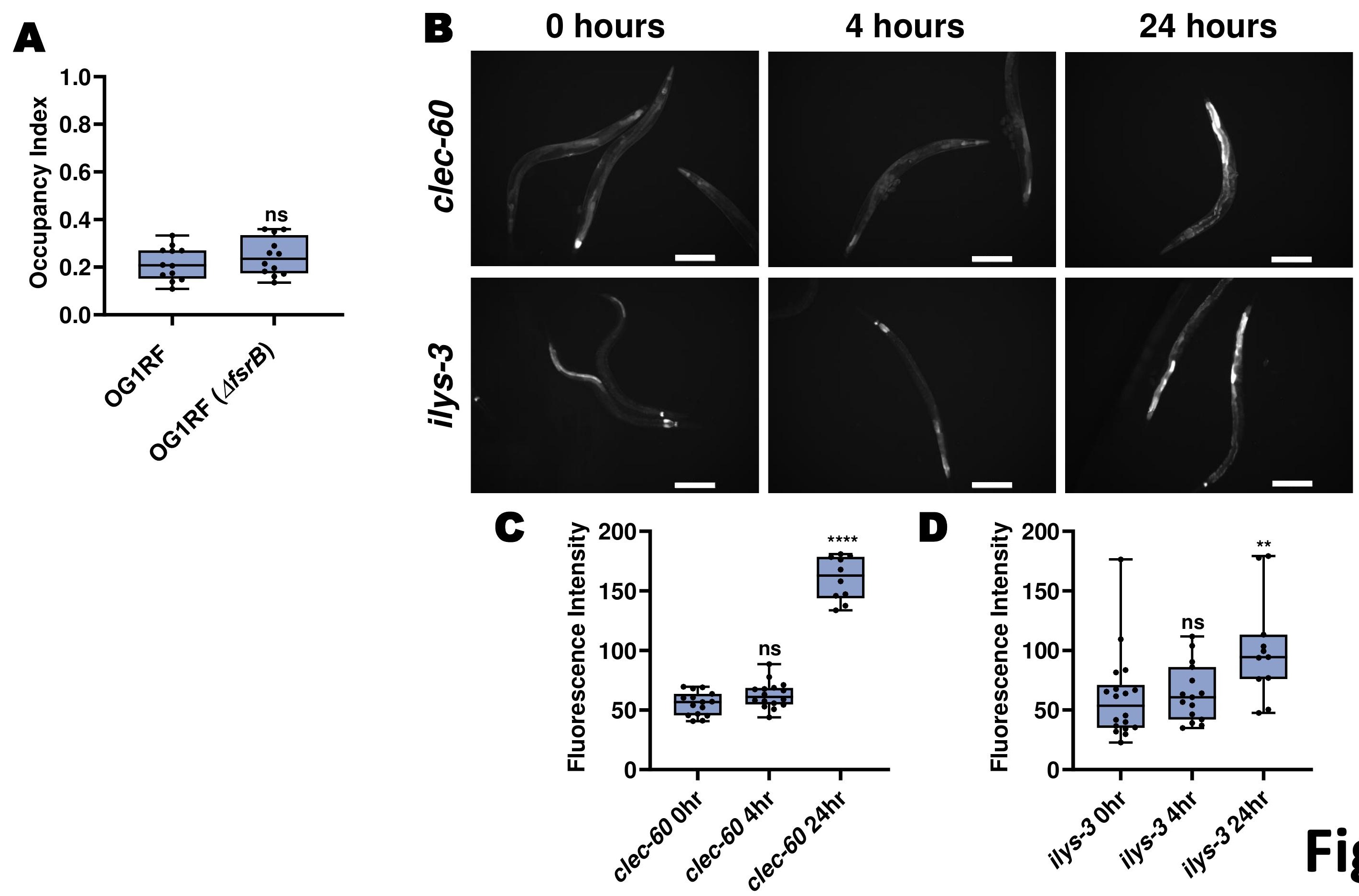

D.

Figure 3 
$\mathbf{A} \times{ }^{1.0} 7 \quad$ B

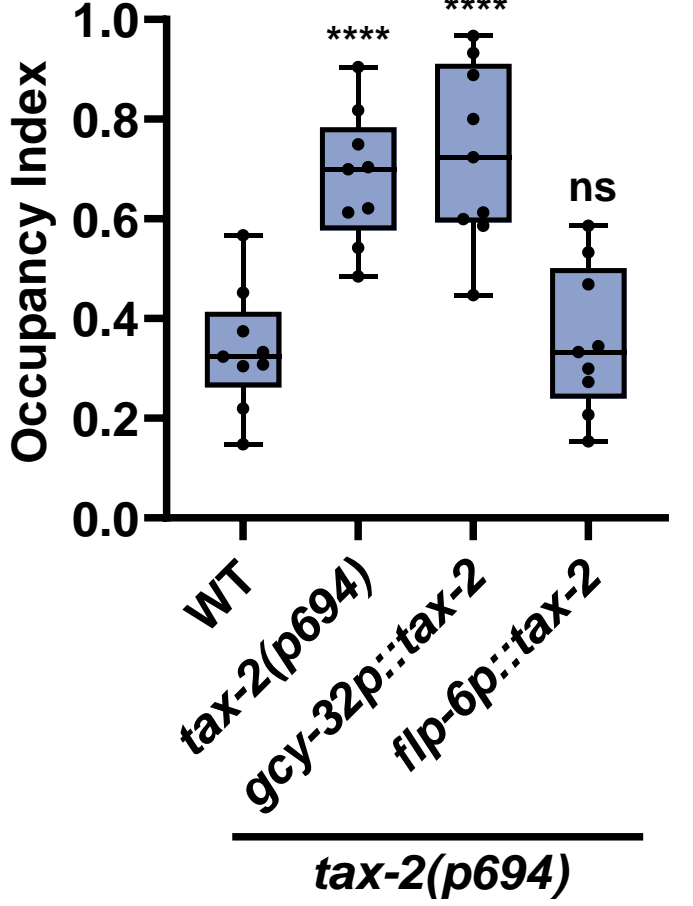

C
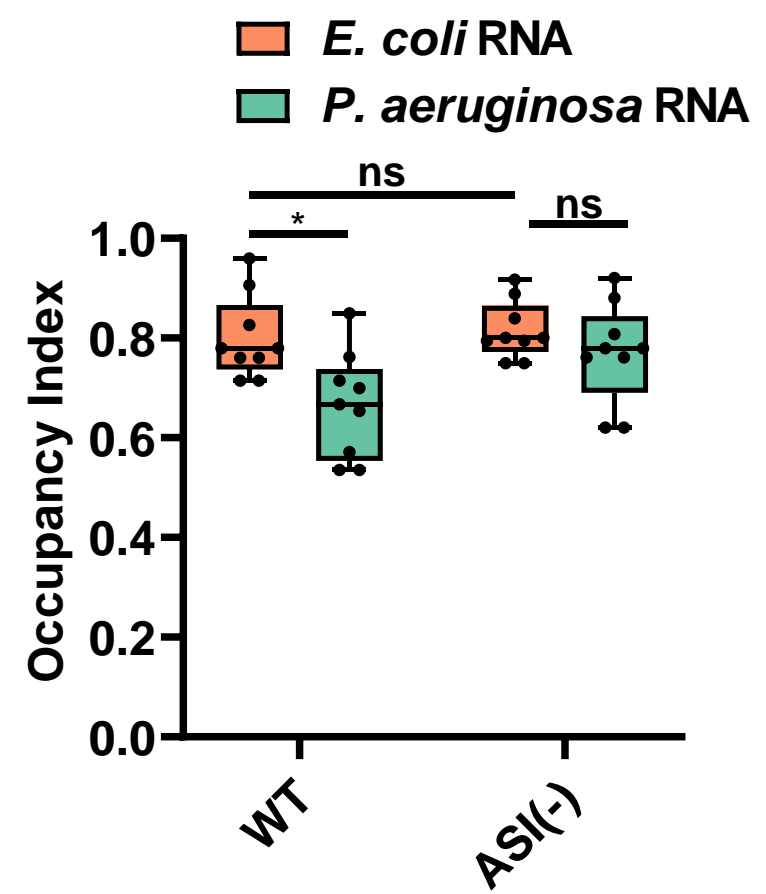

$\square$ E. faecalis

$\square$ P. aeruginosa

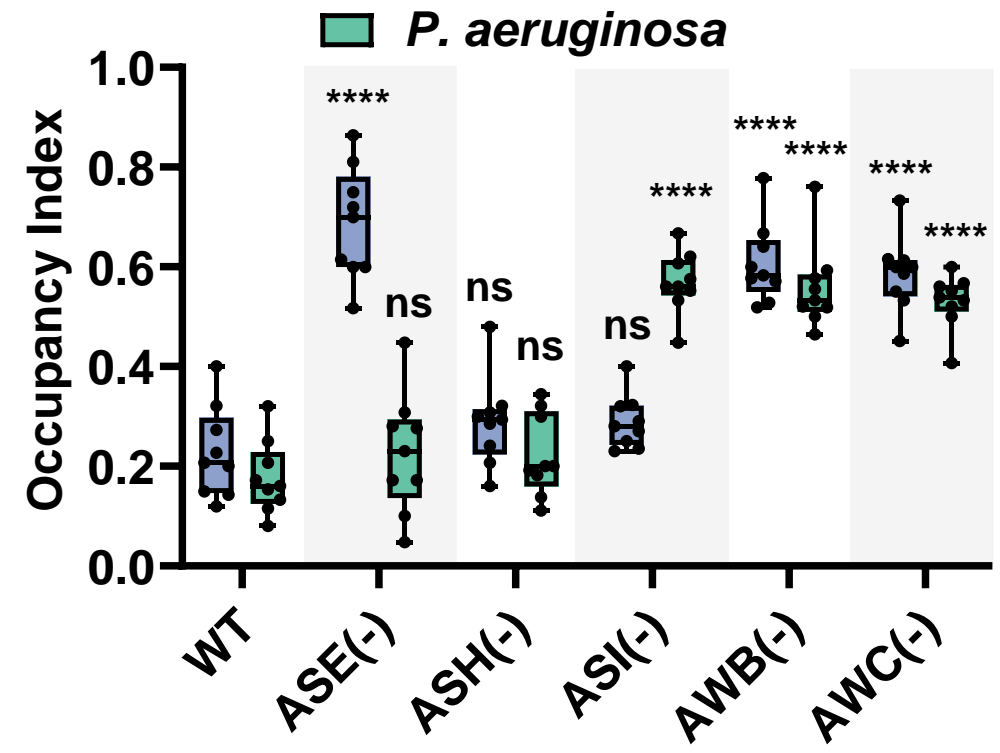

D

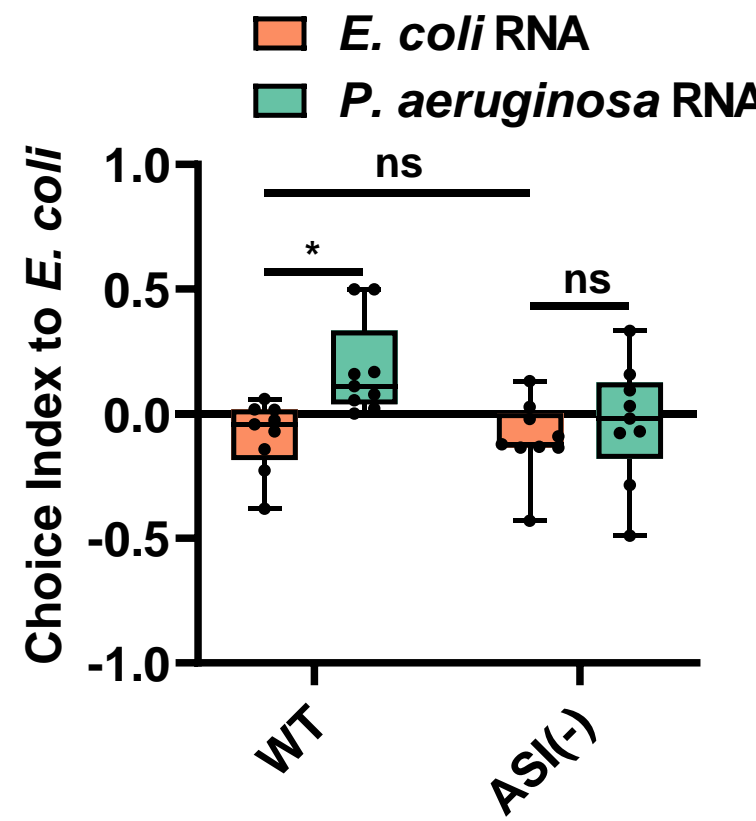

E

P. aeruginosa

Exposure

Intestine-wide Distention

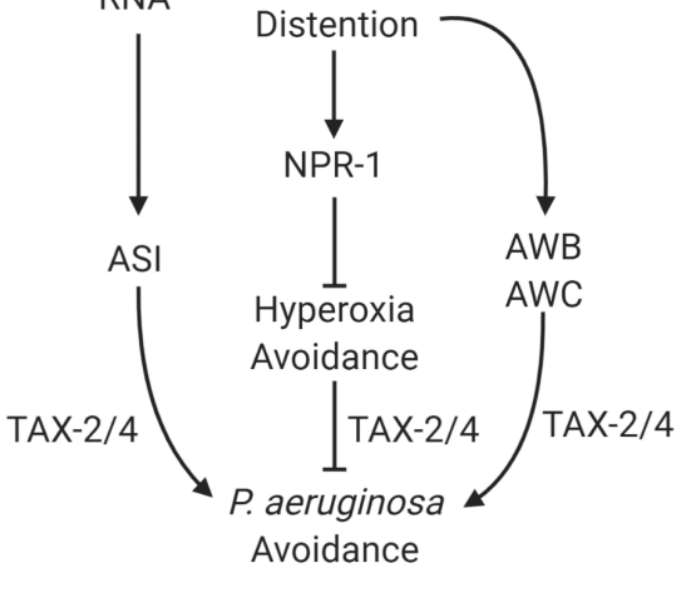

E. faecalis

Exposure

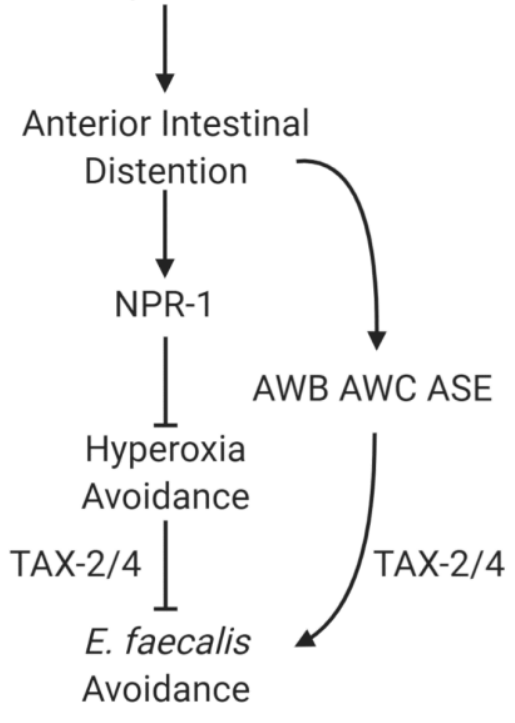

Figure 5 
B

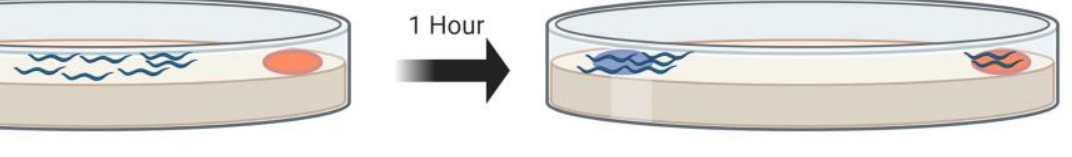

$\square$ Naive

$\square$ Trained

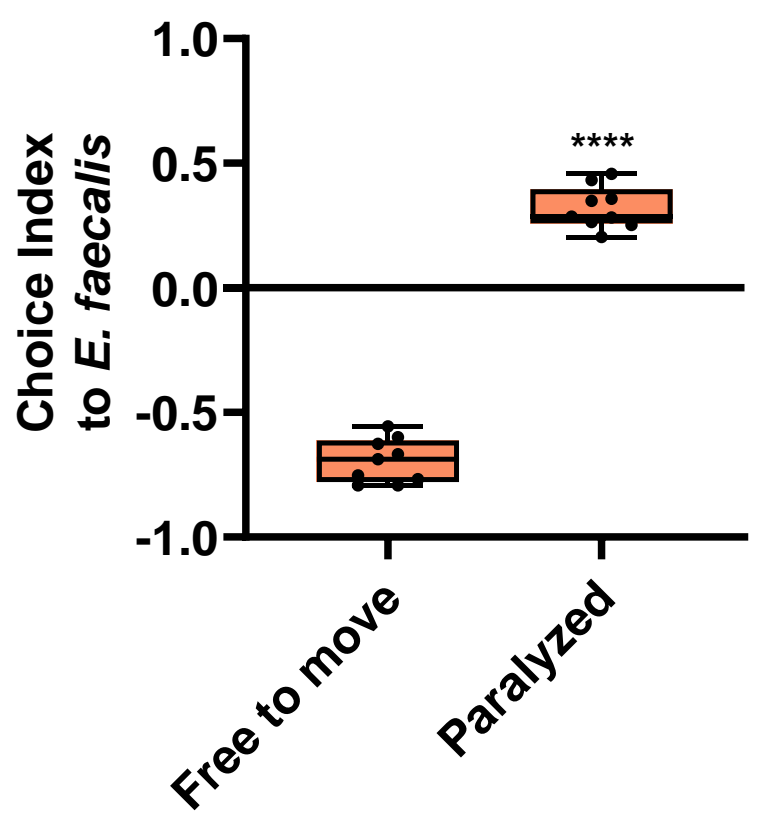

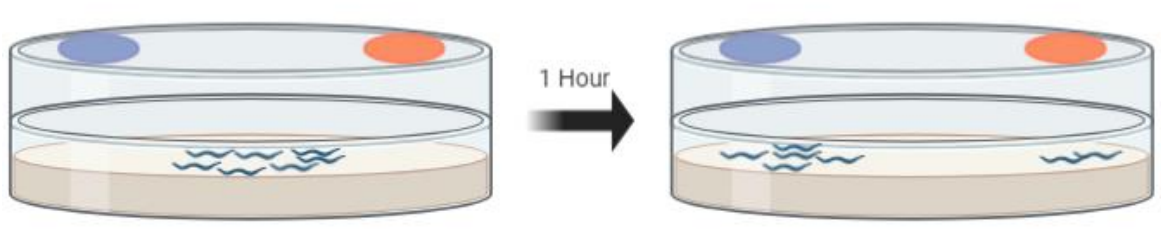

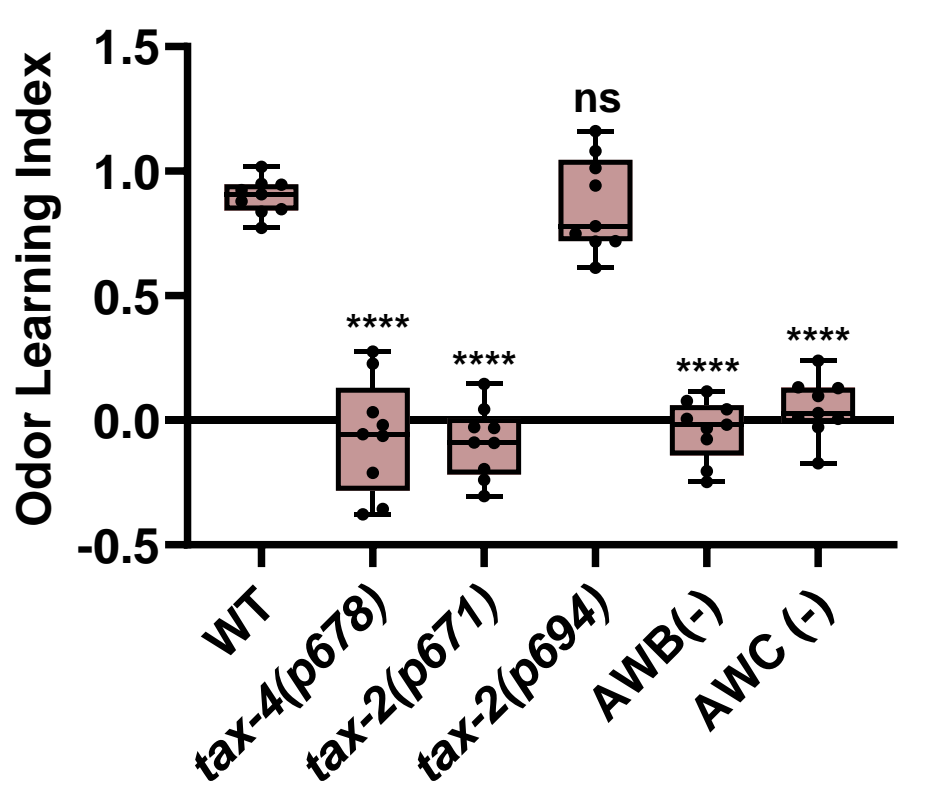

Figure 6 
A

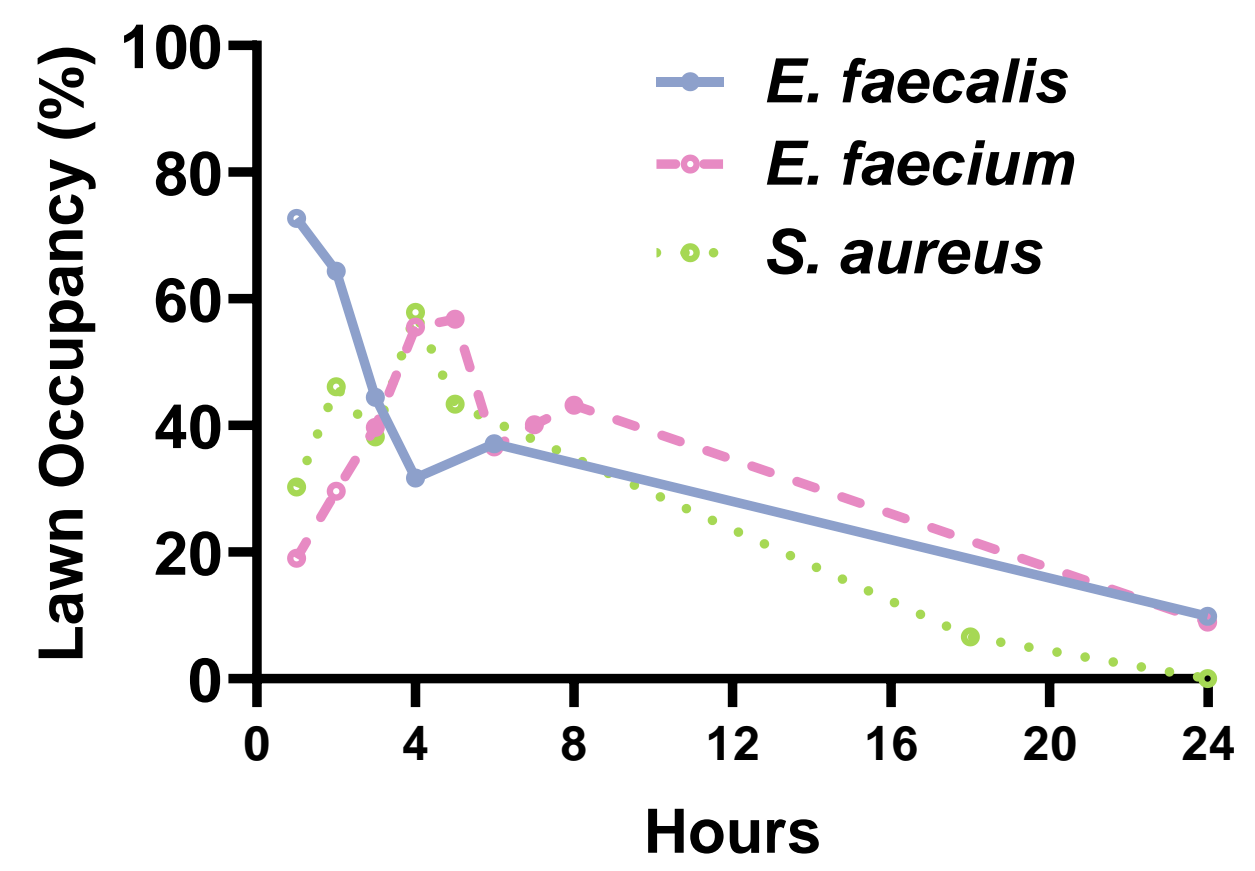

B

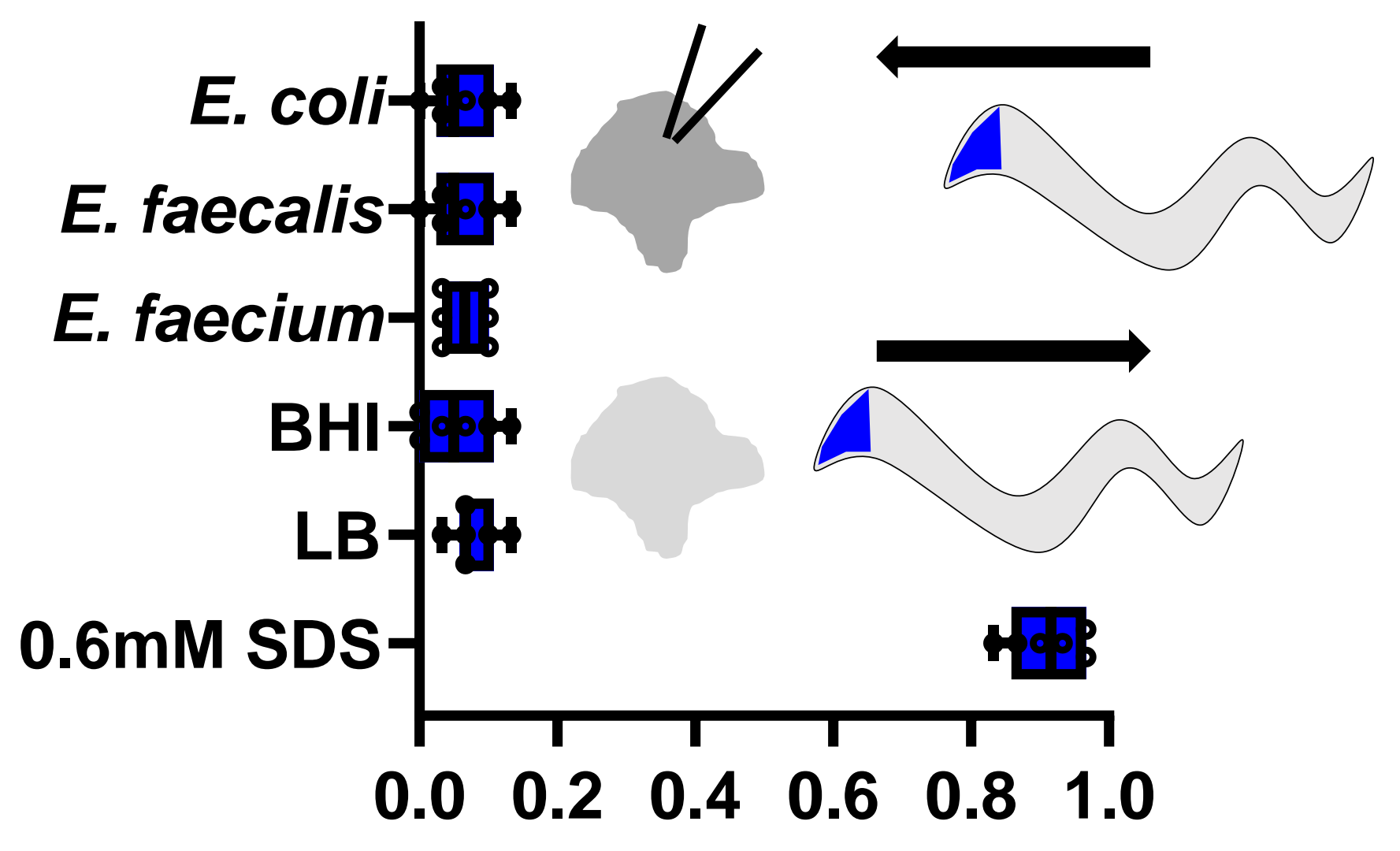

Response Index

Figure 1 - Supplement 1 
A

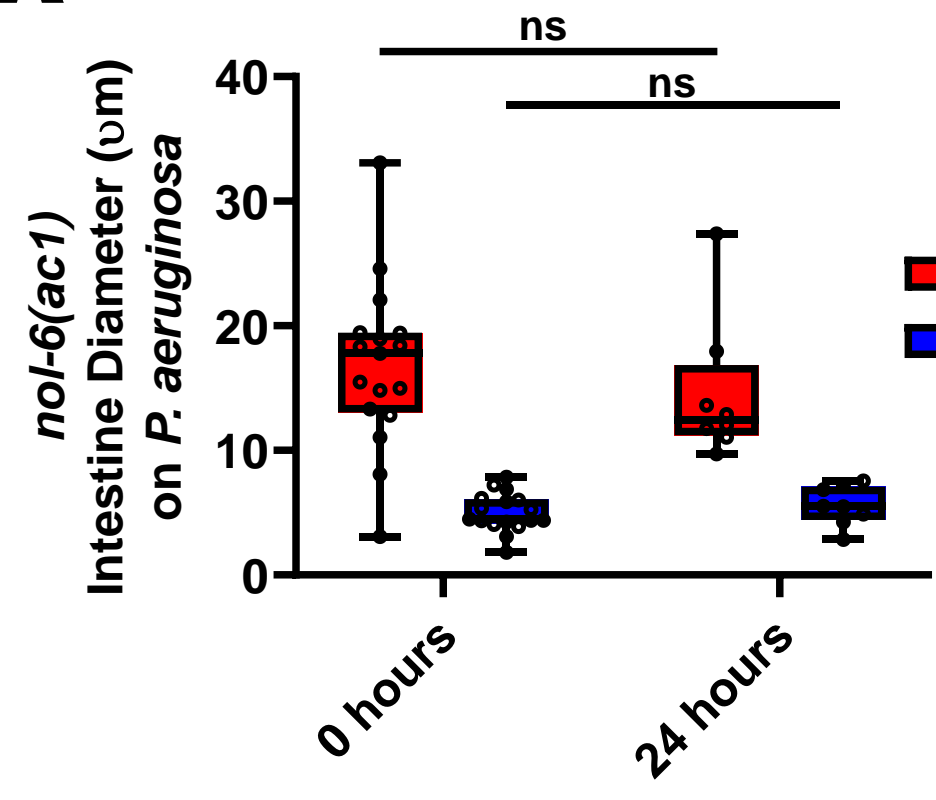

C

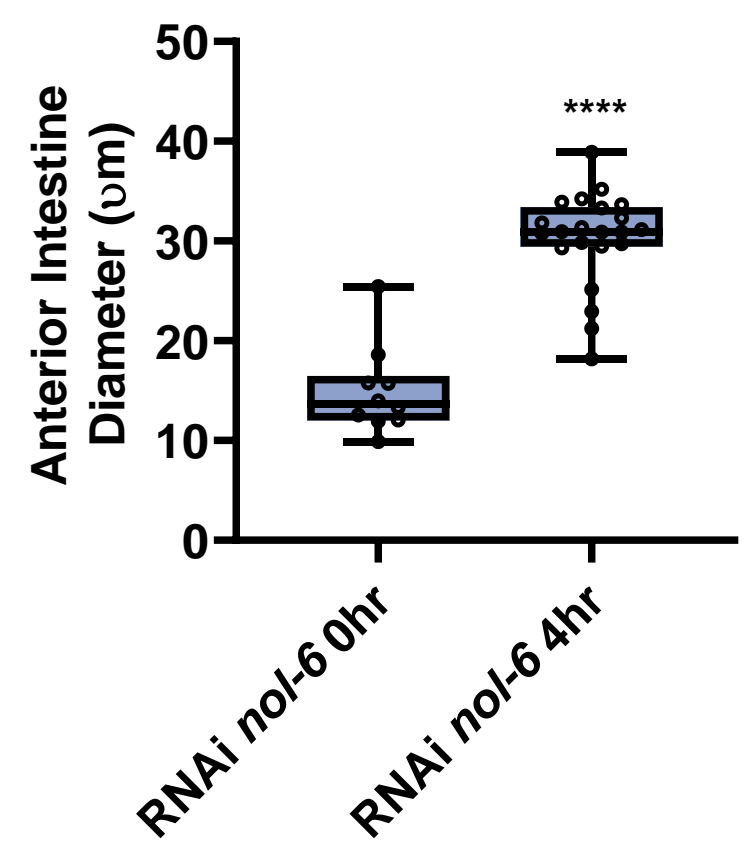

Anterior

a Medial

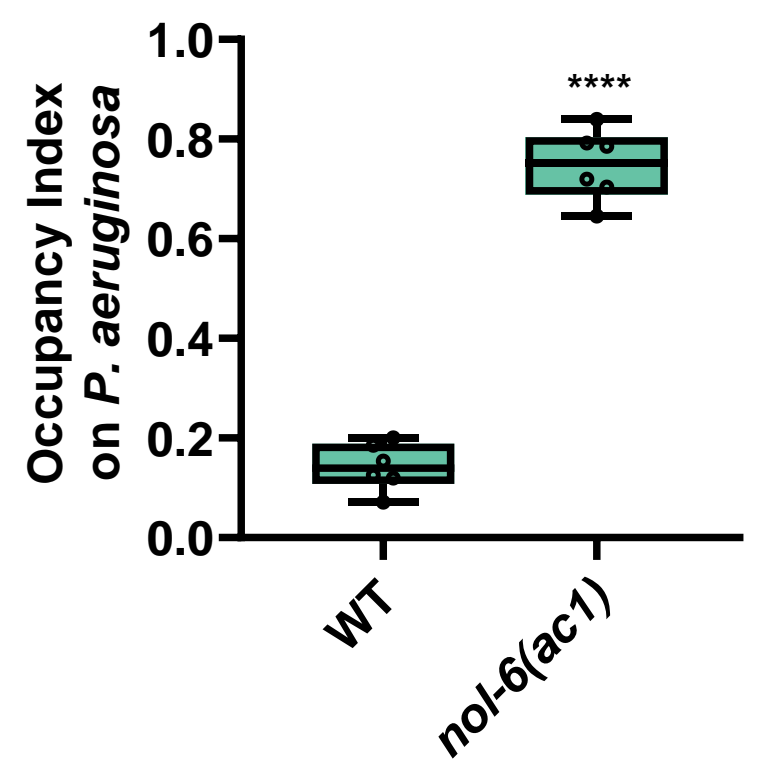

D

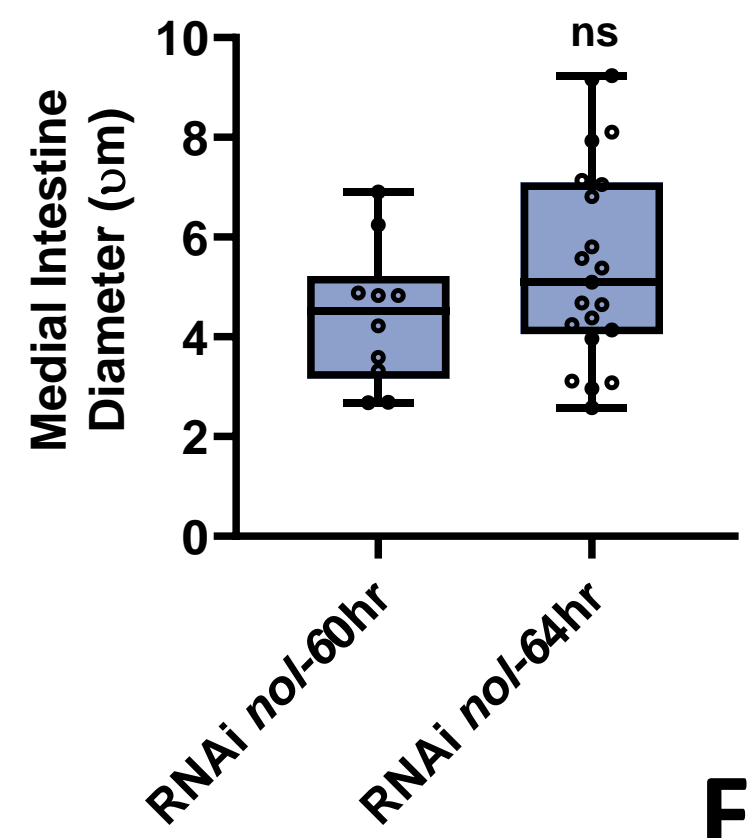

Figure 2 - Supplement 1 
A

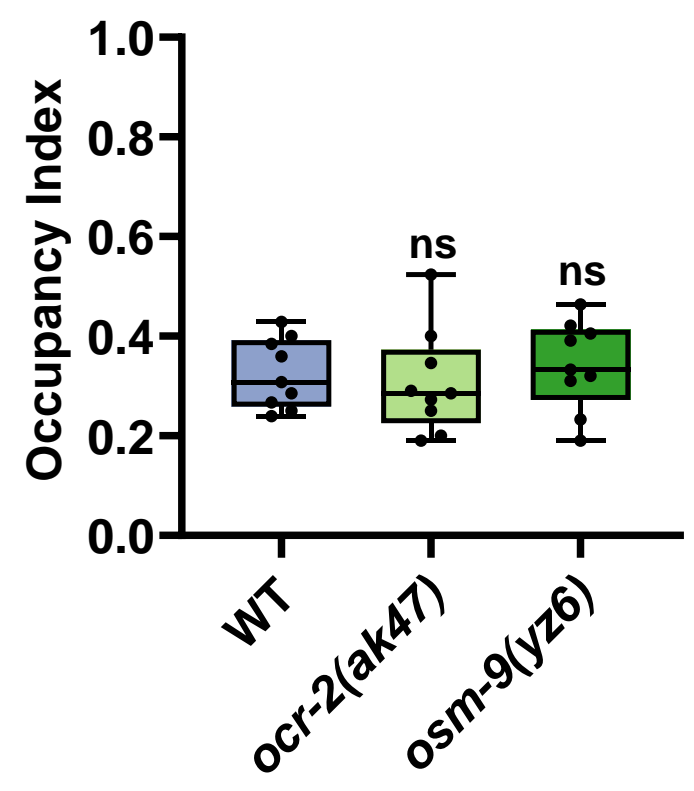

B

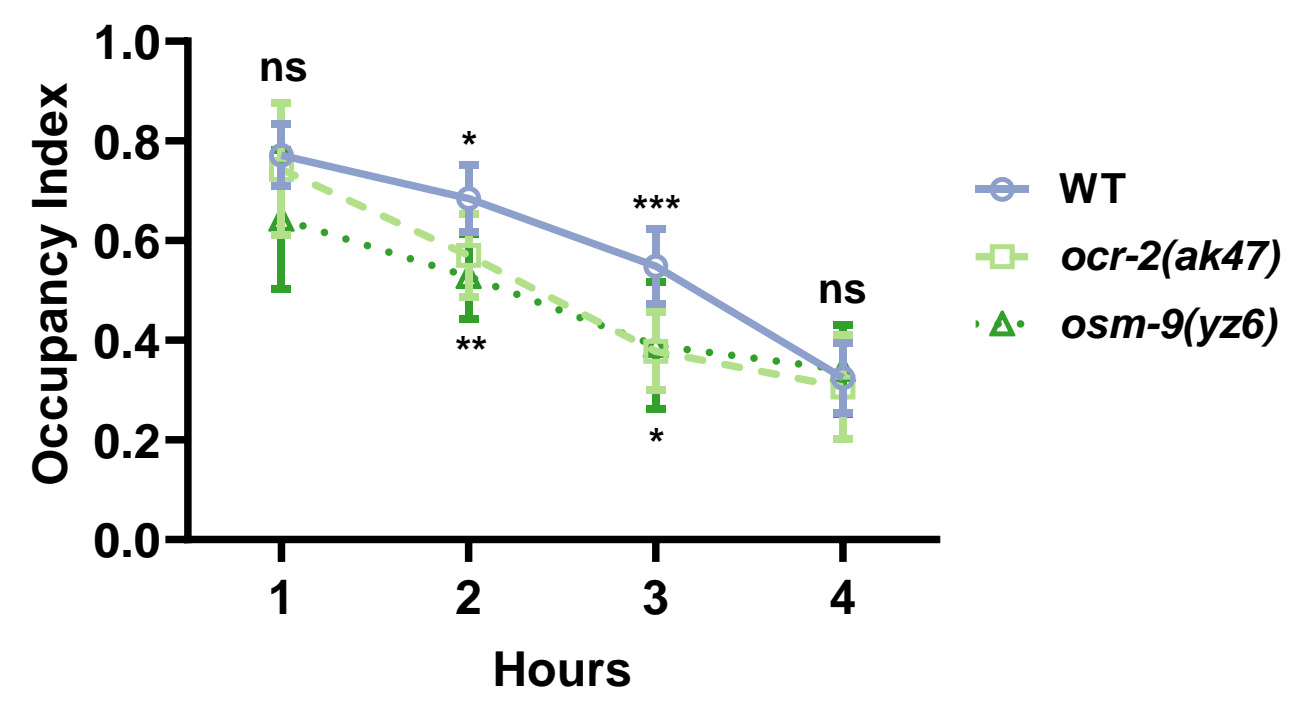




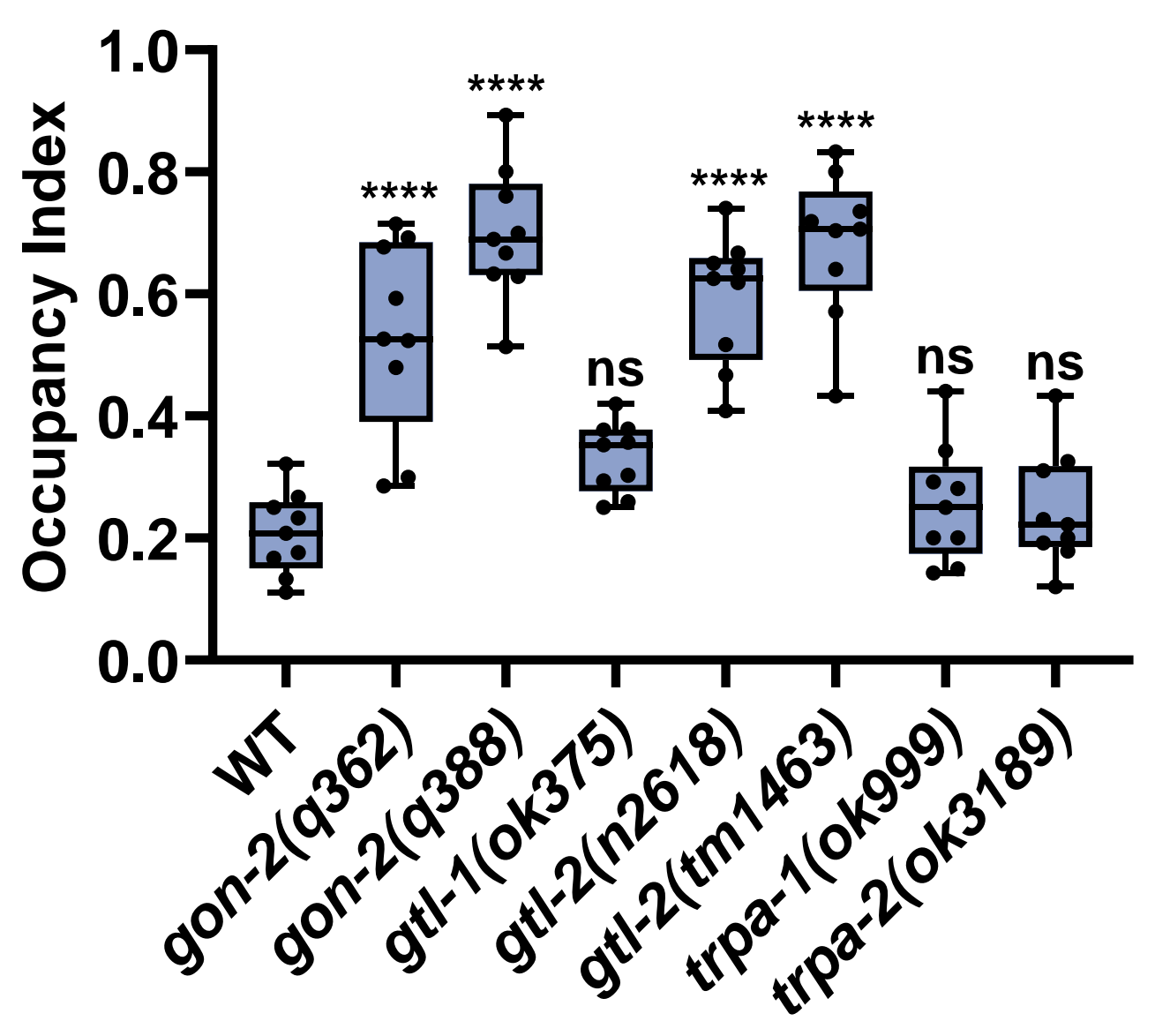

Figure 7 - Supplement 1 
bioRxiv preprint doi: https://doi.org/10.1101/2020.12.18.423492; this version posted December 19, 2020. The copyright holder for this preprint (which was not certified by peer review) is the author/funder, who has granted bioRxiv a license to display the preprint in perpetuity. It is made available under aCC-BY-NC-ND 4.0 International license.

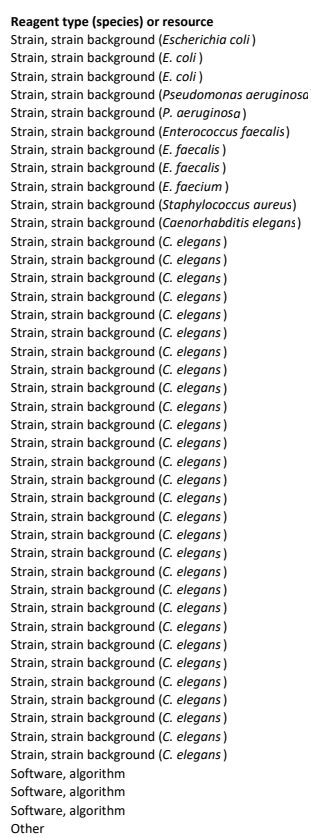

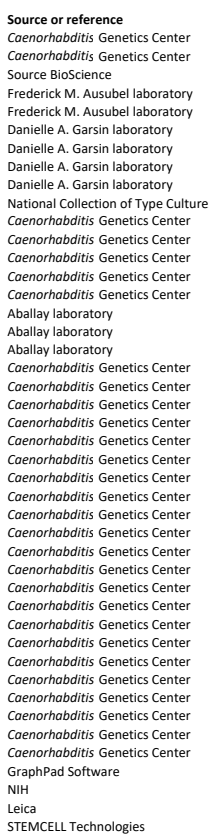

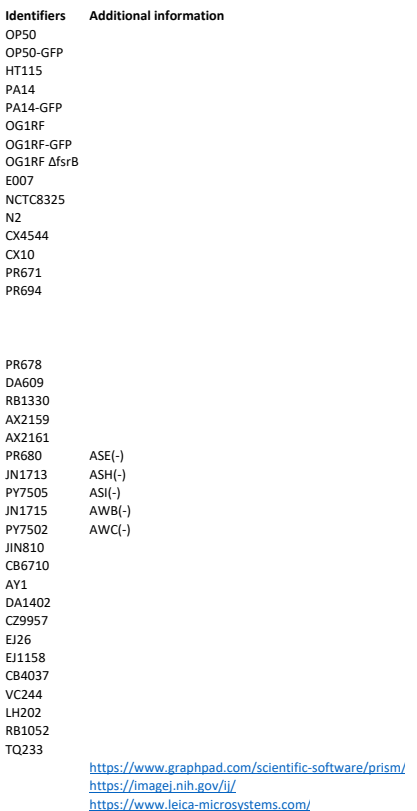

CAT\# 27310 https://www.stemcell.com/products/hypoxia-incubator-chamber.html 